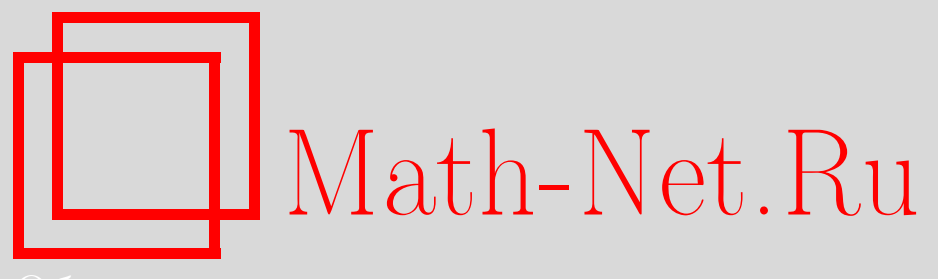

В. И. Богачев, М. Рёкнер, С. В. Шапошников, Глобальная регулярность и оценки решений параболических уравнений, Теория вероятн. и ее примен., 2005, том 50, выпуск 4, 652-674

DOI: https://doi.org/10.4213/tvp124

Использование Общероссийского математического портала MathNet.Ru подразумевает, что вы прочитали и согласны с пользовательским соглашением

http://www . mathnet.ru/rus/agreement

Параметры загрузки:

IP : 3.81 .55 .215

26 апреля 2023 г., 15:16:06

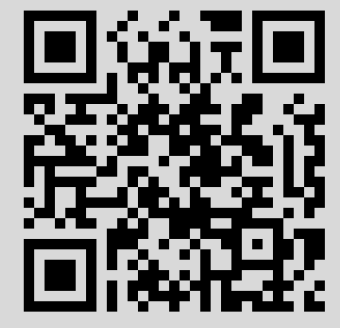



ШАПОШНИКОВ С. В.*

\section{ГЛОБАЛЬНАЯ РЕГУЛЯРНОСТЬ И ОЦЕНКИ РЕШЕНИЙ ПАРАБОЛИЧЕСКИХ УРАВНЕНИЙ ${ }^{1)}$}

Для заданного параболического оператора второго порядка

$$
L u(t, x):=\frac{\partial u(t, x)}{\partial t}+a^{i j}(t, x) \partial_{x_{i}} \partial_{x_{j}} u(t, x)+b^{i}(t, x) \partial_{x_{i}} u(t, x),
$$

рассматривается слабое параболическое уравнение $L^{*} \mu=0$ для борелевских вероятностных мер на $(0,1) \times \mathbf{R}^{d}$. Уравнение понимается как равенство

$$
\int_{(0,1) \times \mathbf{R}^{d}} L u d \mu=0
$$

для всех гладких функций $u$ с компактным носителем в $(0,1) \times \mathbf{R}^{d}$. Это уравнение выполнено для переходных вероятностей диффузионного процесса, ассоциированного с $L$. Показано, что при широких предположениях $\mu$ имеет вид $\mu=\varrho(t, x) d t d x$, где функция $x \mapsto \varrho(t, x)$ является соболевской, функция $\left|\nabla_{x} \varrho(x, t)\right|^{2} / \varrho(t, x)$ интегрируема по Лебегу на $[0, \tau] \times \mathbf{R}^{d}$ и $\varrho \in L^{p}\left([0, \tau] \times \mathbf{R}^{d}\right)$ для всех $p \in[1,+\infty)$ и $\tau<1$. Более того, дано достаточное условие равномерной ограниченности $\varrho$ на $[0, \tau] \times \mathbf{R}^{d}$.

Ключевые слова и фразы: параболическое уравнение для мер, переходные вероятности, регулярность решений параболических уравнений, оценки решений параболических уравнений.

\section{1. Введение и обозначения}

Цель этой работы - дать эффективные условия глобальной соболевской регулярности и интегрируемости плотностей решений параболических уравнений вида

$$
L^{*} \mu=0
$$

* Московский государственный университет им. М.В. Ломоносова, механикоматематический факультет, Ленинские горы, 119992 Москва, Россия; e-mail: sfb1@mathematic.uni-bielefeld.de

** Department of Mathematics, Purdue, University, 150 N. University Str., West Lafayette, IN 47907-2067, USA

1) Работа выполнена проектами РФФИ 04-01-00748, HШ 1758.2003.1, DFG 436 RUS 113/343/0(R), INTAS 03-51-5018 и SFB 701 при университете Билефельда. 
для борелевских мер $\mu$ на $(0,1) \times \mathbf{R}^{d}$. Такие уравнения недавно исследовались в [1]-[4]. Здесь $L$ - параболический оператор второго порядка

$$
L u(t, x):=\frac{\partial u(t, x)}{\partial t}+a^{i j}(t, x) \partial_{x_{i}} \partial_{x_{j}} u(t, x)+b^{i}(t, x) \partial_{x_{i}} u(t, x),
$$

а интерпретация нашего уравнения состоит в следующем. Будем говорить, что борелевская вероятностная мера $\mu$ на $(0,1) \times \mathbf{R}^{d}$, представленная в виде $\mu(d t d x)=\mu_{t}(d x) d t$ посредством семейства борелевских мер $\left(\mu_{t}\right)_{t \in[0,1)}$ на $\mathbf{R}^{d}$, удовлетворяет слабому параболическому уравнению (1.1), если функции $a^{i j}$ и $b^{i}$ интегрируемы на каждом компактном множестве в $(0,1) \times \mathbf{R}^{d}$ относительно меры $\mu=\mu_{t} d t$ и для каждого $u \in C_{0}^{\infty}\left((0,1) \times \mathbf{R}^{d}\right)$ имеем

$$
\int_{(0,1) \times \mathbf{R}^{d}} L u d \mu=\int_{0}^{1} \int_{\mathbf{R}^{d}} L u(t, x) \mu_{t}(d x) d t=0 .
$$

Будем говорить, что $\mu$ удовлетворяет начальному условию $\mu_{0}=\nu$ при $t=0$, если $\nu$ - борелевская мера на $\mathbf{R}^{d}$ и

$$
\lim _{t \rightarrow 0} \int_{\mathbf{R}^{d}} \zeta(x) \mu_{t}(d x)=\int_{\mathbf{R}^{d}} \zeta(x) \nu(d x)
$$

для всех $\zeta \in C_{0}^{\infty}\left(\mathbf{R}^{d}\right)$.

Такие же определения вводятся в случае, когда $\mathbf{R}^{d}$ заменяется открытым множеством $\Omega \subset \mathbf{R}^{d}$ или открытым множеством в римановом многообразии.

Уравнение (1.1) выполнено для переходных вероятностей диффузионного процесса, управляемого стохастическим дифференциальным уравнением

$$
d \xi_{t}=\sqrt{2 A\left(t, \xi_{t}\right)} d w_{t}+b\left(t, \xi_{t}\right) d t
$$

при условии, что такая диффузия существует, а коэффициенты $A$ и $b$ удовлетворяют некоторым условиям. Однако (1.1) может рассматриваться независимо от всяких вероятностных предположений. Более того, изучение этого уравнения в чисто аналитической постановке может быть полезно для построения ассоциированной диффузии (см. [4]).

Наш основной результат утверждает, что плотность $\varrho$ всякого решения обладает тем свойством, что функция $\varrho(t, \cdot)$ является соболевской на пространстве $\mathbf{R}^{d}$, а функция $\left|\nabla_{x} \varrho(t, x)\right|^{2} / \varrho(t, x)$ интегрируема на $[0, \tau] \times \mathbf{R}^{d}$ при условии, что функции $|b|$ и $\ln (|x|+1)$ входят в $L^{2}(\mu)$, коэффициент $A$ равномерно ограничен, равномерно обратим и равномерно липшицев по $x$, а начальное распределение $\mu_{0}=\varrho(0, \cdot) d x$ имеет конечную энтропию. Предположения относительно $A$ можно ослабить, если $b$ обладает некоторой дополнительной локальной интегрируемостью. Дано конструктивное условие в терминах функций Ляпунова, 
обеспечивающее квадратичную интегрируемость $|b|$ и $\ln (|x|+1)$ относительно решения $\mu$. Основной результат позволяет показать, что $\varrho$ входит во все $L^{p}\left([0, \tau] \times \mathbf{R}^{d}\right)$ при $\tau<1$, если $\sup _{t}\|b(t, \cdot)\|_{L^{d}\left(\mu_{t}\right)}<\infty$ и $\varrho(0, \cdot) \in L^{p}\left(\mathbf{R}^{d}\right)$ для всех $p \geqslant 1$. Если же $|b| \in L^{\beta}(\mu)$ при некотором $\beta>d+2$ и $\varrho(0, \cdot) \in L^{\infty}\left(\mathbf{R}^{d}\right)$, то плотность $\varrho$ равномерно ограничена на $[0, \tau] \times \mathbf{R}^{d}$ при $\tau<1$. С помощью этого утверждения получены поточечные оценки сверху вида $\varrho(t, x) \leqslant \Phi(x)^{-1}$. Отметим, что в отличие от многих известных результатов о глобальной ограниченности решений здесь не требуется, чтобы коэффициент сноса $b$ был диссипативным или потенциальным.

Аналогичные результаты в эллиптическом случае были получены в [5]-[10]. Можно было бы рассмотреть эллиптический случай как ситуацию, когда решение и коэффициенты не зависят от времени. Тогда наш параболический результат не покрывает эллиптический, так как начальное распределение (которое в этом случае совпадает с решением) должно иметь конечную энтропию, а последнее предположение не может быть полностью устранено в параболическом случае. С другой стороны, разумный параболический аналог эллиптического результата мог бы быть таким: интегрируемость $|\varrho|^{2} / \varrho$ на $\left[\tau_{1}, \tau_{2}\right] \times \mathbf{R}^{d}$ для всякого отрезка $\left[\tau_{1}, \tau_{2}\right] \subset(0,1)$ без ограничений на начальное распределение. Пока мы не преуспели в исследовании этой второй возможности. Конечно, если для некоторого $\tau>0$ мера $\mu_{\tau}$ имеет конечную энтропию, то наши предположения выполнены на $[\tau, 1]$.

Наш результат будет применен в готовящейся работе о проблеме единственности для параболических уравнений для мер. Он может быть полезен также в изучении переходных вероятностей диффузионных процессов и в динамике Нельсона (см. [11]-[15]).

Пусть $W^{p, 1}\left(\mathbf{R}^{d}\right)$ обозначает соболевское пространство функций, принадлежащих $L^{p}\left(\mathbf{R}^{d}\right)$ вместе с их обобщенными частными производными. Это пространство наделяется стандартной нормой

$$
\|f\|_{W^{p, 1}}:=\|f\|_{p}+\|\nabla f\|_{p}
$$

где $\|\cdot\|_{p}$ обозначает $L^{p}\left(\mathbf{R}^{d}\right)$-норму скалярных или векторных функций. Символ $L^{p, q}$, где $1 \leqslant p, q<\infty$, будет использоваться для пространства всех измеримых функций $f$ на $[0,1] \times \mathbf{R}^{d}$ с конечной нормой

$$
\|f\|_{p, q}:=\left(\int_{0}^{1}\left(\int_{\mathbf{R}^{d}}|f(t, x)|^{p} d x\right)^{q / p} d t\right)^{1 / q} .
$$

Пространство $L^{p, \infty}$, где $1 \leqslant p<\infty$, состоит из измеримых функций $f$ на $[0,1] \times \mathbf{R}^{d}$ c $t \mapsto\|f(t, \cdot)\|_{L^{p}\left(\mathbf{R}^{d}\right)} \in L^{\infty}[0,1]$. Для аналогичных пространств функций на $[0, \tau] \times \mathbf{R}^{d}$ используются обозначения $L^{p, q}\left([0, \tau] \times \mathbf{R}^{d}\right)$. Наконец, $\mathbf{H}^{p, q}\left([0, \tau] \times \mathbf{R}^{d}\right)$ обозначает пространство всех измеримых функций $f$ 
на $[0, \tau] \times \mathbf{R}^{d}$ с конечной нормой

$$
\|f\|_{\mathbf{H}^{p, q}\left([0, \tau] \times \mathbf{R}^{d}\right)}:=\left(\int_{0}^{\tau}\|f(t, \cdot)\|_{W^{p, 1}}^{q} d t\right)^{1 / q} .
$$

Для упрошения обозначений градиент функции $u$ на $(0,1) \times \mathbf{R}^{d}$ относительно аргумента из $\mathbf{R}^{d}$ обозначается через $\nabla u$, т.е.

$$
\nabla u(t, x):=\nabla_{x} u(t, x)=\left(\partial_{x_{1}} u(t, x), \ldots, \partial_{x_{d}} u(t, x)\right) .
$$

Мы используем стандартное правило суммирования по повторяющимся индексам, например, $\partial_{x_{i}} a^{i j}:=\sum_{i=1}^{d} \partial_{x_{i}} a^{i j}, a^{i j} \partial_{x_{i}} \partial_{x_{j}} u:=$ $\sum_{i, j=1}^{d} a^{i j} \partial_{x_{i}} \partial_{x_{j}} u$.

Будем говорить, что неотрицательная мера $\mu_{0}$ на $\mathbf{R}^{d}$ имеет конечную энтропию, если $\mu_{0}=\varrho_{0} d x$ и $\varrho_{0} \ln \varrho_{0} \in L^{1}\left(\mathbf{R}^{d}\right)$, где мы полагаем $0 \ln 0:=0$. Энтропией $\varrho_{0}$ называется интеграл от $\varrho_{0} \ln \varrho_{0}$.

В [3] было показано, что если коэффициент $A$ невырожден, то мера $\mu$ абсолютно непрерывна относительно меры Лебега на $(0,1) \times \mathbf{R}^{d}$. Соответствующая плотность будет обозначаться через $\varrho$.

Достаточное условие существования решения в классе вероятностных мер состоит в следующем (см. работу [2], которая улучшает [1]). Коэффициенты $a^{i j}$ и $b^{i}$ - такие борелевские функции на $[0,1] \times \mathbf{R}^{d}$, что $A(t, x)=\left(a^{i j}(t, x)\right)$ является неотрицательной симметричной матрицей, причем существует такое $p>d+2$, что для каждого шара $B$ и всех $i, j \leqslant d$ имеем

(C1) $\inf _{(t, x) \in[0,1] \times B} \operatorname{det} A(t, x) \geqslant M_{1}(B)>0$ и выполняется неравенство $\sup _{t \in[0,1]}\left\|a^{i j}(t, \cdot)\right\|_{W^{p, 1}(B)} \leqslant M_{2}(B)$,

$(\mathrm{C} 2) \sup _{t \in[0,1]}\left\|b^{i}(t, \cdot)\right\|_{L^{p}(B)} \leqslant M_{3}(B)$.

Если выполнены (C1) и (C2) и существует такая неотрицательная функция $V$ на $\mathbf{R}^{d}$, что $\lim _{|x| \rightarrow+\infty} V(x)=+\infty$ и $L V \leqslant c_{1} V+c_{2}$ с некоторыми постоянными $c_{1}$ и $c_{2}$, то решение $\mu=\mu_{t}(d x) d t$ с вероятностными мерами $\mu_{t}$ существует для каждого начального распределения $\mu_{0}$, причем функция $t \mapsto \int_{\mathbf{R}^{d}} \zeta(x) \mu_{t}(d x)$ непрерывна на $[0,1)$ для всех $\zeta \in C_{0}^{\infty}\left(\mathbf{R}^{d}\right)$. Более того, если $V \in L^{1}\left(\mu_{0}\right)$, то $\int_{0}^{1} \int_{\mathbf{R}^{d}} V(x) \mu_{t}(d x) d t<\infty$. Кроме того, если $L V \leqslant c_{2}$, то $\int_{\mathbf{R}^{d}} V(x) \mu_{t}(d x) \leqslant c_{2}$ для почти всех $t$. Например, если коэффициент $A$ равномерно ограничен и

$$
\langle b(t, x), x\rangle \leqslant k_{1}|x|^{2}+k_{2},
$$

то возьмем функцию $V(x)=\ln \left(|x|^{2}+1\right)$. Это дает оценку $L V \leqslant$ const, значит, интегралы от $\ln (|x|+1)$ относительно $\mu_{t}$ равномерно ограничены, если $\ln (|x|+1) \in L^{1}\left(\mu_{0}\right)$. Если коэффициент $A$ равномерно ограничен и

$$
\langle b(t, x), x\rangle \leqslant k_{1}|x|^{2} \ln (|x|+1)+k_{2}
$$

то положим $V(x)=\left|\ln \left(|x|^{2}+1\right)\right|^{2}$ и получим $L V \leqslant c_{1} V+c_{2}$, значит, является $\mu$-интегрируемой функция $|\ln (|x|+1)|^{2}$, если она $\mu_{0}$-интегрируема. 
Наша основная оценка будет установлена в двух случаях, соответствующих двум различным подходам:

1) когда (C1) заменяется более сильным предположением и (C2) заменяется условием, что $|b|, \ln \max (|x|, 1) \in L^{2}(\mu)$,

2) условие $|b|, \ln \max (|x|, 1) \in L^{2}(\mu)$ накладывается в дополнение к (C1), (C2) и некоторому глобальному условию на $A$.

Положим

$$
\Theta_{A}(t, x):=\sum_{j=1}^{d}\left|\sum_{i=1}^{d} \partial_{x_{i}} a^{i j}(t, x)\right| .
$$

\section{2. Оценки логарифмических градиентов}

Наш первый основной результат устанавливает квадратичную интегрируемость логарифмического градиента $\mu$, т.е. отображения $\nabla \varrho / \varrho$, относительно $\mu$. Если $\varrho(t, \cdot) \in W_{\text {loc }}^{1,1}$, то мы используем следуюшее соглашение: $\nabla \varrho(t, x) / \varrho(t, x):=0$ при $\varrho(t, x)=0$.

Теорема 2.1. Предположим, что $\mu$, где каждая $\mu_{t}$ есть вероятностная мера, удовлетворяет (1.1), (1.3). Пусть

(i) отображение $A$ равномерно ограничено и $A(t, x) \geqslant \alpha \cdot I$ для некоторой постоянной $\alpha>0$, а функции $x \mapsto a^{i j}(t, x)$ липшичевы $c$ постоянной $\lambda$,

(ii) $|b| \in L^{2}(\mu)$.

Предположим также, что функиия $\Lambda(x):=\ln \max (|x|, 1)$ входит в $L^{2}(\mu)$ (что имеет место, если, например, $\langle b(t, x), x\rangle \leqslant C_{1}|x|^{2} \Lambda(x)+C_{2}$ с некоторыми постоянными $\left.C_{1} u C_{2} u \Lambda \in L^{2}\left(\mu_{0}\right)\right)$. Eсли $\mu_{0}$ имеет конечную энтропию, то $\mu_{t}=\varrho(t, \cdot) d x$, где $\varrho(t, \cdot) \in W^{1,1}\left(\mathbf{R}^{d}\right)$, причем для каждого $\tau<1$ имеем

$$
\int_{0}^{\tau} \int_{\mathbf{R}^{d}} \frac{|\nabla \varrho(t, x)|^{2}}{\varrho(t, x)} d x d t<\infty .
$$

$B$ частности, имеем $\sqrt{\varrho} \in \mathbf{H}^{2,2}\left([0, \tau] \times \mathbf{R}^{d}\right), \varrho \in L^{d /(d-2), 1}\left([0, \tau] \times \mathbf{R}^{d}\right)$, если $d>2$, и $\varrho \in L^{s, 1}\left([0, \tau] \times \mathbf{R}^{d}\right)$ для всех $s \in[1, \infty)$ nрu $d=2$.

Eсли интегральь $\int_{\mathbf{R}^{d}} \varrho(t, x) \Lambda(x) d x$ ограничень при $t \rightarrow 1$ (что выполнено, например, если $\langle b(t, x), x\rangle \leqslant C_{1}|x|^{2}+C_{2}$ с некоторыми постоянными $C_{1}$ и $C_{2}$ и $\left.\Lambda \in L^{1}\left(\mu_{0}\right)\right)$, то (2.1) верно с $\tau=1$.

Д о к а з а т е л ь с т в о. Воспользуемся следующим фактом (см., например, лемму 2.1 в работе [6]): если даны две неотрицательные функции $f_{1}, f_{2} \in L^{1}\left(\mathbf{R}^{d}\right)$, то для всякой измеримой функции $\psi$ такой, что $|\psi|^{2} f_{1} \in L^{1}\left(\mathbf{R}^{d}\right)$, имеем

$$
\int_{\mathbf{R}^{d}} \frac{\left|\left(\psi f_{1}\right) * f_{2}\right|^{2}}{f_{1} * f_{2}} d x \leqslant \int_{\mathbf{R}^{d}}|\psi|^{2} f_{1} d x \int_{\mathbf{R}^{d}} f_{2} d x,
$$

где $\left|\left(\psi f_{1}\right) * f_{2}(x)\right|^{2} /\left(f_{1} * f_{2}(x)\right):=0$, если $f_{1} * f_{2}(x)=0$. 
Для функции $w \in C_{0}^{\infty}\left(\mathbf{R}^{d}\right)$ положим

$$
\varrho * w(t, x):=\int_{\mathbf{R}^{d}} w(x-y) \varrho(t, y) d y, \quad x \in \mathbf{R}^{d} .
$$

Здесь и далее свертки берутся всегда относительно аргумента из $\mathbf{R}^{d}$. Из уравнения (1.2) и включения $|b| \in L^{2}(\mu)$ вытекает, что в соболевском смысле выполнено равенство

$$
\partial_{t}(\varrho * w)=\left(a^{i j} \varrho\right) * \partial_{x_{i}} \partial_{x_{j}} w-\left(b^{i} \varrho\right) * \partial_{x_{i}} w
$$

Мы будем иметь дело с версией $\varrho * w$ (обозначаемой тем же символом), заданной формулой

$$
\varrho * w(t, x):=\varrho * w(0, x)+\int_{0}^{t} v(s, x) d s,
$$

где $v$ - правая часть (2.3). Так как $|b| \in L^{2}(\mu)$ и функции $a^{i j}$ ограничены, то $v \in L^{1}\left([0,1] \times \mathbf{R}^{d}\right)$. Поэтому функция $\varrho * w$ абсолютно непрерывна по $t$ на $[0,1]$ и входит в класс $C_{b}^{\infty}\left(\mathbf{R}^{d}\right)$ по $x$. При почти каждом $t$ указанная версия совпадает при всех $x$ с исходной версией, заданной сверткой. Ниже важно также то, что это верно для $t=0$. Поскольку исходная версия не превосходит $\sup _{x}|w(x)|$, то это же верно и для новой версии при почти всех $t$, а тогда и поточечно в силу непрерывности по $t$. Из условий (i) и (ii) легко усмотреть, что сказанное, включая (2.3), остается верным также для функций $w_{\varepsilon}(x)=\varepsilon^{-d} g(x / \varepsilon)$, где $g$ - стандартная гауссовская плотность и $\varepsilon \in(0,1)$. Ниже в качестве $\varepsilon$ берем только числа вида $1 / n, n \in \mathbf{N}$. Положим

$$
\varrho_{\varepsilon}:=\varrho * w_{\varepsilon}, \quad f_{\varepsilon}(t, x):=\varrho_{\varepsilon}(t, x)+\varepsilon \max (1,|x|)^{-d-1},
$$

причем в качестве $\varrho * w_{\varepsilon}$ берем версию, указанную в (2.4). Так как функция $\varrho \Lambda$ интегрируема, то сколь угодно близко к 1 найдутся такие $\tau$, что

$$
\int_{\mathbf{R}^{d}} \varrho(\tau, x) \Lambda(x) d x<\infty
$$

Число $\tau$, для которого выполнено (2.5), можно взять так, что для каждого $\varepsilon=1 / n$ наша версия $\varrho_{\varepsilon}(\tau, x)$ будет совпадать со сверткой $\varrho(\tau, \cdot) * w_{\varepsilon}(x)$ при всех $x$. Тогда легко проверяемое неравенство $\ln \max (|x+y|, 1) \leqslant \ln \max (|x|, 1)+|y|$ дает

$$
\begin{aligned}
& \int_{\mathbf{R}^{d}} f_{\varepsilon}(\tau, x) \Lambda(x) d x \leqslant \int_{\mathbf{R}^{d}} \varrho_{\varepsilon}(\tau, x) \Lambda(x) d x+\varepsilon \int_{\mathbf{R}^{d}} \max (|x|, 1)^{-d-1} \Lambda(x) d x \\
& \leqslant \int_{\mathbf{R}^{d}} \varrho(\tau, x) \Lambda(x) d x+\int_{\mathbf{R}^{d}}|y| w_{\varepsilon}(y) d y+\varepsilon \int_{\mathbf{R}^{d}} \max (|x|, 1)^{-d-1} \Lambda(x) d x \\
& \leqslant \int_{\mathbf{R}^{d}} \varrho(\tau, x) \Lambda(x) d x+\varepsilon M_{1},
\end{aligned}
$$


где $M_{1}$ - число, не зависящее от $\varepsilon$. В силу (2.3) имеем

$$
\begin{aligned}
& \int_{0}^{\tau} \int_{\mathbf{R}^{d}} \partial_{t}\left(\varrho * w_{\varepsilon}\right) \ln f_{\varepsilon} d x d t \\
& \quad=\int_{0}^{\tau} \int_{\mathbf{R}^{d}}\left[\left(a^{i j} \varrho\right) * \partial_{x_{i}} \partial_{x_{j}} w_{\varepsilon}-\left(b^{i} \varrho\right) * \partial_{x_{i}} w_{\varepsilon}\right] \ln f_{\varepsilon} d x d t
\end{aligned}
$$

ибо $\left|\ln f_{\varepsilon}\right| \leqslant c_{1}+c_{2} \Lambda$ с некоторыми постоянными $c_{1}$ и $c_{2}$, а функции $\left(\left(b^{i} \varrho\right) * \partial_{x_{i}} w_{\varepsilon}\right) \Lambda$ и $\left(\left(a^{i j} \varrho\right) * \partial_{x_{i}} \partial_{x_{j}} w_{\varepsilon}\right) \Lambda$ интегрируемы на $(0,1) \times \mathbf{R}^{d}$. Действительно, так как $\varrho *\left|\partial_{x_{i}} w_{\varepsilon}\right|>0$, то

$$
\left(\left(b^{i} \varrho\right) * \partial_{x_{i}} w_{\varepsilon}\right) \Lambda=\left(\left(b^{i} \varrho\right) * \partial_{x_{i}} w_{\varepsilon}\right)\left(\varrho *\left|\partial_{x_{i}} w_{\varepsilon}\right|\right)^{-1 / 2}\left(\varrho *\left|\partial_{x_{i}} w_{\varepsilon}\right|\right)^{1 / 2} \Lambda \text {. }
$$

Тогда $\left(\left(b^{i} \varrho\right) * \partial_{x_{i}} w_{\varepsilon}\right)\left(\varrho *\left|\partial_{x_{i}} w_{\varepsilon}\right|\right)^{-1 / 2} \in L^{2}\left((0,1) \times \mathbf{R}^{d}\right)$ в силу $(2.2)$ и включения $|b| \in L^{2}(\mu)$. Кроме того, $\left(\varrho *\left|\partial_{x_{i}} w_{\varepsilon}\right|\right)^{1 / 2} \Lambda \in L^{2}\left((0,1) \times \mathbf{R}^{d}\right)$ в силу оценки $|\ln \max (|x+y|, 1)|^{2} \leqslant 4+2|\ln \max (|x|, 1)|^{2}+2|\ln \max (|y|, 1)|^{2}$ и таких же выкладок, как и в (2.6). Аналогичным образом проверяется интегрируемость $\left[\left(a^{i j} \varrho\right) * \partial_{x_{i}} \partial_{x_{j}} w_{\varepsilon}\right] \Lambda$ на $(0,1) \times \mathbf{R}^{d}$. Заметим, что можно проинтегрировать по частям в правой части (2.7). Действительно,

$\int_{0}^{\tau} \int_{\mathbf{R}^{d}} \frac{\left|\nabla f_{\varepsilon}\right|^{2}}{f_{\varepsilon}} d x d t \leqslant 2 \int_{0}^{\tau} \int_{\mathbf{R}^{d}} \frac{\left|\nabla \varrho_{\varepsilon}\right|^{2}}{\varrho_{\varepsilon}} d x d t+2 \varepsilon(d+1)^{2} \int_{\{|x| \geqslant 1\}}|x|^{-d-3} d x$,

что конечно в силу $(2.2)$, поскольку $\nabla \varrho_{\varepsilon}=\left(\nabla w_{\varepsilon}\right) * \varrho$ и $\left|\nabla w_{\varepsilon}\right|^{2} / w_{\varepsilon} \in$ $L^{1}\left(\mathbf{R}^{d}\right)$. Кроме того, $\left[\left(b^{i} \varrho\right) * w_{\varepsilon}\right] \varrho_{\varepsilon}^{-1 / 2} \in L^{2}\left((0,1) \times \mathbf{R}^{d}\right)$ также в силу $(2.2)$ и включения $|b| \in L^{2}(\mu)$. Аналогично имеем $\left[\left(a^{i j} \varrho\right) * \partial_{x_{i}} w_{\varepsilon}\right] \varrho_{\varepsilon}^{-1 / 2} \in$ $L^{2}\left((0,1) \times \mathbf{R}^{d}\right)$. Поскольку $f_{\varepsilon}>\varrho_{\varepsilon}$, то $\partial_{x_{i}} f_{\varepsilon}\left(\partial_{x_{j}}\left[\left(a^{i j} \varrho\right) * w_{\varepsilon}\right]-\left(b^{i} \varrho\right) * w_{\varepsilon}\right) f_{\varepsilon}^{-1} \in$ $L^{1}\left(\mathbf{R}^{d}\right)$. Следовательно,

$\int_{0}^{\tau} \int_{\mathbf{R}^{d}} \partial_{t} \varrho_{\varepsilon} \ln f_{\varepsilon} d x d t=-\int_{0}^{\tau} \int_{\mathbf{R}^{d}} \frac{\partial_{x_{i}} f_{\varepsilon}}{f_{\varepsilon}}\left(\partial_{x_{j}}\left[\left(a^{i j} \varrho\right) * w_{\varepsilon}\right]-\left(b^{i} \varrho\right) * w_{\varepsilon}\right) d x d t$.

Подынтегральное выражение слева можно записать как $\partial_{t}\left(f_{\varepsilon} \ln f_{\varepsilon}\right)-\partial_{t} \varrho_{\varepsilon}$. Так как интегралы от $\varrho_{\varepsilon}(\tau, x)$ и $\varrho_{\varepsilon}(0, x)$ по $x$ равны 1, мы видим, что левая часть (2.8) равна

$$
L_{\varepsilon}:=\int_{\mathbf{R}^{d}}\left[f_{\varepsilon}(\tau, x) \ln f_{\varepsilon}(\tau, x)-f_{\varepsilon}(0, x) \ln f_{\varepsilon}(0, x)\right] d x .
$$

Поскольку $f_{\varepsilon}(\tau, \cdot) \ln f_{\varepsilon}(\tau, \cdot) \in L^{1}\left(\mathbf{R}^{d}\right)$ ввиду (2.6) и оценки $\left|\ln f_{\varepsilon}\right| \leqslant$ $c_{1}+c_{2} \Lambda$, то $f_{\varepsilon}(0, \cdot) \ln f_{\varepsilon}(0, \cdot) \in L^{1}\left(\mathbf{R}^{d}\right)$. Нам нужна оценка снизу на $L_{\varepsilon}$. С этой целью заметим, что ввиду выпуклости функции $s \mapsto s \ln s$ на $(0,+\infty)$ и неравенства Йенсена мы имеем

$$
\begin{gathered}
\int_{\mathbf{R}^{d}} f_{\varepsilon}(0, x) \ln f_{\varepsilon}(0, x) d x \leqslant \int_{\mathbf{R}^{d}} \varrho_{\varepsilon}(0, x) \ln \left(2 \varrho_{\varepsilon}(0, x)\right) d x \\
\quad+\int_{\mathbf{R}^{d}} \varepsilon \max (|x|, 1)^{-d-1} \ln \left(2 \varepsilon \max (|x|, 1)^{-d-1}\right) d x
\end{gathered}
$$




$$
\begin{aligned}
& \leqslant \ln 2+\int_{\mathbf{R}^{d}} \varrho_{\varepsilon}(0, x) \ln \varrho_{\varepsilon}(0, x) d x+\varepsilon \ln 2 \int_{\mathbf{R}^{d}} \max (|x|, 1)^{-d-1} d x \\
& \leqslant \ln 2+\int_{\mathbf{R}^{d}} \varrho_{0}(x) \ln \varrho_{0}(x) d x+\varepsilon \ln 2 \int_{\mathbf{R}^{d}} \max (|x|, 1)^{-d-1} d x=: M(\varepsilon) .
\end{aligned}
$$

С другой стороны, (2.6) дает

$$
\begin{gathered}
\int_{\mathbf{R}^{d}} f_{\varepsilon}(\tau, x) \ln f_{\varepsilon}(\tau, x) d x \geqslant-(d+1) \int_{\mathbf{R}^{d}} f_{\varepsilon}(\tau, x) \Lambda(x) d x \\
\geqslant-(d+1) \int_{\mathbf{R}^{d}} \varrho(\tau, x) \Lambda(x) d x-\varepsilon M_{1}(d+1)=:-K(\varepsilon) .
\end{gathered}
$$

Заметим, что для всякой ограниченной борелевской функции $а$ на $(0,1) \times \mathbf{R}^{d}$, которая липшицева по второму аргументу с константой Липшица $\lambda$, для каждого $j$ имеем

$$
\begin{aligned}
& \partial_{x_{j}}\left[(a \varrho) * w_{\varepsilon}\right](t, x)=a(t, x) \partial_{x_{j}} \varrho_{\varepsilon}(t, x) \\
& \quad+\int_{\mathbf{R}^{d}} \partial_{x_{j}} w_{\varepsilon}(x-y)[a(t, y)-a(t, x)] \varrho(t, y) d y
\end{aligned}
$$

и

$$
\begin{aligned}
& \left|\int_{\mathbf{R}^{d}} \partial_{x_{j}} w_{\varepsilon}(x-y)[a(t, y)-a(t, x)] \varrho(t, y) d y\right| \\
& \quad \leqslant \lambda \int_{\mathbf{R}^{d}}\left|\partial_{x_{j}} w_{\varepsilon}(x-y)\right||y-x| \varrho(t, y) d y \\
& \quad \leqslant \lambda \int_{\mathbf{R}^{d}} \varepsilon^{-d} \frac{|x-y|^{2}}{\varepsilon^{2}} g\left(\frac{x-y}{\varepsilon}\right) \varrho(t, y) d y=\lambda\left(\varrho * q_{\varepsilon}\right)(t, x),
\end{aligned}
$$

где $q_{\varepsilon}(x):=w_{\varepsilon}(x)|x / \varepsilon|^{2}, x \in \mathbf{R}^{d}$. Отметим для дальнейшего, что при выводе $(2.9)$ и (2.10) мы не использовали $\mu$-интегрируемость $\Lambda^{2}$ и существование энтропии для $\mu_{0}$. Используя $(2.8)$ и $(2.9)$, получаем

$$
\begin{aligned}
& \int_{0}^{\tau} \int_{\mathbf{R}^{d}} a^{i j} \frac{\partial_{x_{i}} f_{\varepsilon}}{f_{\varepsilon}} \partial_{x_{j}} f_{\varepsilon} d x d t \\
& =\int_{0}^{\tau} \int_{\mathbf{R}^{d}} \frac{\partial_{x_{i}} f_{\varepsilon}}{f_{\varepsilon}}\left[\left(b^{i} \varrho\right) * w_{\varepsilon}+\varepsilon a^{i j} \partial_{x_{j}} \frac{1}{\max (|x|, 1)^{d+1}}\right] d x d t \\
& \quad-\int_{0}^{\tau} \int_{\mathbf{R}^{d}}\left(\frac{\partial_{x_{i}} f_{\varepsilon}(t, x)}{f_{\varepsilon}(t, x)} \int_{\mathbf{R}^{d}} \partial_{x_{j}} w_{\varepsilon}(x-y)\right. \\
& \left.\times\left[a^{i j}(t, y)-a^{i j}(t, x)\right] \varrho(t, y) d y\right) d x d t-L_{\varepsilon} .
\end{aligned}
$$

Правая часть этого равенства не превосходит

$$
\begin{aligned}
& \left(\int_{0}^{\tau} \int_{\mathbf{R}^{d}} \frac{\left|\nabla f_{\varepsilon}\right|^{2}}{f_{\varepsilon}} d x d t\right)^{1 / 2}\left[\left(\int_{0}^{\tau} \int_{\mathbf{R}^{d}} \frac{\sum_{i=1}^{d}\left[\left(b^{i} \varrho\right) * w_{\varepsilon}\right]^{2}}{f_{\varepsilon}} d x d t\right)^{1 / 2}+\varepsilon M C_{d}\right] \\
& \quad+d^{3 / 2} \lambda\left(\int_{0}^{\tau} \int_{\mathbf{R}^{d}} \frac{\left|\nabla f_{\varepsilon}\right|^{2}}{f_{\varepsilon}} d x d t\right)^{1 / 2}\left(\int_{0}^{\tau} \int_{\mathbf{R}^{i}} \frac{\left(\varrho * q_{\varepsilon}\right)^{2}}{f_{\varepsilon}} d x d t\right)^{1 / 2} \\
& \quad+M(\varepsilon)+K(\varepsilon)
\end{aligned}
$$


где $M=\sup _{t, x}\|A(t, x)\|$ и $C_{d}$ - интеграл от $(d+1)^{2}|x|^{-d-3}$ по множеству $\{|x| \geqslant 1\}$. В силу $(2.2)$ имеем

$$
\begin{gathered}
\int_{0}^{\tau} \int_{\mathbf{R}^{d}} \frac{\left[\left(b^{i} \varrho\right) * w_{\varepsilon}\right]^{2}}{f_{\varepsilon}} d x d t \leqslant \int_{0}^{\tau} \int_{\mathbf{R}^{d}}\left|b^{i}\right|^{2} d \mu, \quad 1 \leqslant i \leqslant d, \\
\int_{0}^{\tau} \int_{\mathbf{R}^{d}} \frac{\left(\varrho * q_{\varepsilon}\right)^{2}}{f_{\varepsilon}} d x d t \leqslant \gamma:=\int_{\mathbf{R}^{d}}|x|^{4} g(x) d x .
\end{gathered}
$$

Поскольку $A \geqslant \alpha \cdot I$, приходим к оценке

$$
\begin{aligned}
\alpha \int_{0}^{\tau} \int_{\mathbf{R}^{d}} \frac{\left|\nabla f_{\varepsilon}\right|^{2}}{f_{\varepsilon}} d x d t \leqslant & \left(\int_{0}^{\tau} \int_{\mathbf{R}^{d}} \frac{\left|\nabla f_{\varepsilon}\right|^{2}}{f_{\varepsilon}} d x d t\right)^{1 / 2} \\
& \times\left(\|b\|_{2, \mu}+\varepsilon M C_{d}+\lambda d^{3 / 2} \sqrt{\gamma}\right)+M(\varepsilon)+K(\varepsilon),
\end{aligned}
$$

что ввиду неравенства $c \sqrt{x} \leqslant \alpha x / 2+c^{2} /(2 \alpha)$ дает

$$
\begin{aligned}
\int_{0}^{\tau} \int_{\mathbf{R}^{d}} \frac{\left|\nabla f_{\varepsilon}\right|^{2}}{f_{\varepsilon}} d x d t \leqslant & \alpha^{-2}\left(\|b\|_{2, \mu}+\varepsilon M C_{d}+\lambda d^{3 / 2} \sqrt{\gamma}\right)^{2} \\
& +2 \alpha^{-1}(M(\varepsilon)+K(\varepsilon)) .
\end{aligned}
$$

Величины $M(\varepsilon)$ и $K(\varepsilon)$ равномерно ограничены по $\varepsilon$. Полагая $\varepsilon \rightarrow 0$, получаем, что $\sqrt{\varrho(t, \cdot)} \in W^{2,1}\left(\mathbf{R}^{d}\right)$ для почти всех $t \in(0, \tau)$. Значит, $\varrho(t, \cdot) \in W^{1,1}\left(\mathbf{R}^{d}\right)$ для почти всех $t \in(0, \tau)$. Кроме того, интеграл от $|\nabla \varrho|^{2} / \varrho$ не превосходит правой части $(2.11)$ с $\varepsilon=0$. Итак, $\sqrt{\varrho} \in \mathbf{H}^{2,2}\left([0, \tau] \times \mathbf{R}^{d}\right)$. По теореме вложения Соболева имеем $\varrho \in$ $L^{d /(d-2), 1}\left([0, \tau] \times \mathbf{R}^{d}\right)$, если $d>2$, и $\varrho \in L^{s, 1}\left([0, \tau] \times \mathbf{R}^{d}\right)$ для всех $s \in[1, \infty)$ при $d=2$.

Последнее утверждение теоремы ясно из наших рассуждений. Теорема 2.1 доказана.

Из доказательства следует полезная оценка

$$
\begin{aligned}
& \int_{0}^{\tau} \int_{\mathbf{R}^{d}} \frac{|\nabla \varrho|^{2}}{\varrho} d x d t \leqslant \alpha^{-2}\left(\|b\|_{2, \mu}+\lambda d^{3 / 2} \sqrt{\gamma}\right)^{2}+2 \ln 2 \alpha^{-1} \\
& \quad+2 \alpha^{-1} \int_{\mathbf{R}^{d}} \varrho_{0}(x) \ln \varrho_{0}(x) d x+2 \alpha^{-1}(d+1) \int_{\mathbf{R}^{d}} \varrho(\tau, x) \Lambda(x) d x .
\end{aligned}
$$

3 а м е ч ан и е 2.1. Из доказательства ясно, что для энтропии $\varrho_{\varepsilon}(0, x)$ нужна лишь оценка сверху, поэтому вместо интегрируемости $\varrho(0, x) \ln \varrho(0, x)$ достаточно требовать лишь интегрируемость $\varrho(0, x) \max (0, \ln \varrho(0, x))$ (тогда неравенство Йенсена надо применять к функции $s \max (0, \ln s))$. Это приводит к тому, что в оценке $(2.12)$ вместо $\varrho(0, x) \ln \varrho(0, x)$ будет стоять $\varrho(0, x) \max (0, \ln \varrho(0, x))$. Однако полученные оценки и (2.8) показывают, что при сохранении прочих наших условий энтропия $\varrho(0, x)$ все равно оказывается конечной. Если же не требовать $\mu$-интегрируемость $\Lambda$, то ситуация может измениться. Например, 
если $d=1, b=0$ и $a=\frac{1}{2}$, то для всякого начального распределения $\mu_{0}$ решение дается сверткой $\mu_{0} * g_{t}$, где $g_{t}(x)=(2 \pi t)^{-1 / 2} \exp \left(-x^{2} /(2 t)\right)$. Если $\mu_{0}$ имеет такую плотность $\varrho_{0}$, что $\left|\varrho_{0}^{\prime}\right|^{2} / \varrho_{0} \in L^{1}\left(\mathbf{R}^{1}\right)$, но функция $\varrho_{0} \ln \varrho_{0}$ не интегрируема, то решение $\varrho(t, x)$ не имеет энтропии ни при каком $t$, хотя величины $\int\left|\partial_{x} \varrho(t, x)\right|^{2} \varrho(t, x)^{-1} d x$ равномерно ограничены. Этот же пример показывает, что для справедливости оценки (2.1) необходимы какието условия на начальное распределение. Достаточно взять в качестве $\mu_{0}$ дираковскую меру в начале координат. Тогда функция $\left|\partial_{x} \varrho\right|^{2} / \varrho$ не интегрируема на $(0,1) \times \mathbf{R}^{1}$. Было бы интересно найти достаточное условие на $A$ и $b$, обеспечивающее конечную энтропию $\varrho(t, \cdot)$ для $t>0$ и всякого начального условия.

В примере 3.1 ниже и в работе [1] можно найти условия на коэффициенты $A$ и $b$, обеспечивающие включение $|b| \in L^{2}(\mu)$.

Оценку (2.12) можно улучшить при дополнительных предположениях относительно $A$ и $b$. Положим $b_{0}:=\left(b_{0}{ }^{j}\right), b_{0}^{j}=b^{j}-\partial_{x_{i}} a^{i j}$.

Теорема 2.2. Предположим, что н удовлетворяет (1.1), (1.3), аде $\nu=\varrho_{0} d x, \oint у н к ц и я \varrho_{0}$ имеет конечную энтропию и локально гёльдерова. Пусть $A$ и b удовлетворяют (C1) u (C2) с некоторьм $p>d+2$. Предположим, что $\left|A^{-1 / 2} b_{0}\right| \in L^{2}(\mu), \ln (1+|x|) \in L^{4}(\mu) u$

$$
\liminf _{r \rightarrow \infty} \int_{0}^{1} \int_{r \leqslant|x| \leqslant 2 r}\left[r^{-4}\|A(t, x)\|^{2}+r^{-2} \Theta_{A}(t, x)^{2}\right] \mu_{t}(d x) d t=0 .
$$

Тогда $\varrho(t, \cdot) \in W_{\text {loc }}^{p, 1}$ u для почти всех $\tau \in[0,1]$ имеем

$$
\begin{aligned}
\int_{0}^{\tau} \int_{\mathbf{R}^{d}}\left|\frac{\sqrt{A} \nabla \varrho}{\varrho}\right|^{2} d \mu \leqslant & \int_{0}^{\tau} \int_{\mathbf{R}^{d}}\left|A^{-1 / 2} b_{0}\right|^{2} d \mu \\
& +2 \int_{\mathbf{R}^{d}}[\varrho(0, x) \ln \varrho(0, x)-\varrho(\tau, x) \ln \varrho(\tau, x)] d x
\end{aligned}
$$

причем правая часть конечна. При дополнительном предположении, что $A \geqslant \alpha \cdot I$ для некоторого $\alpha>0$, имеем $\sqrt{\varrho} \in \mathbf{H}^{2,2}\left([0, \tau] \times \mathbf{R}^{d}\right) u$ $\varrho \in L^{d /(d-2), 1}\left([0, \tau] \times \mathbf{R}^{d}\right)$, если $d>2, u \varrho \in L^{s, 1}\left([0, \tau] \times \mathbf{R}^{d}\right) \partial л я$ всех $s \in[1, \infty)$ npu $d=2$.

Д о к а з а т е л ь с т в о. Из локальной теории [3] мы знаем, что $\mu$ обладает непрерывной положительной плотностью $\varrho$ такой, что для каждого шара $B$ и каждого отрезка $\left[t_{1}, t_{2}\right]$ в $(0,1)$ имеем $\|\varrho(t, \cdot)\|_{W^{p, 1}(B)} \in$ $L^{p}\left[t_{1}, t_{2}\right]$. Пусть $B_{j}$ обозначает замкнутый шар радиуса $j$ с центром в начале координат. Зафиксируем такую функцию $\zeta \in C_{0}^{\infty}\left(\mathbf{R}^{d}\right)$, что $\zeta(x)=1$, если $|x| \leqslant 1$ и $\zeta(x)=0$, если $|x|>2$. Положим $\zeta_{j}(x):=\zeta(x / j)$. Для малых $\varepsilon>0$ и больших $k>0$ положим

$$
\varrho_{k, \varepsilon}:=\min \left(k, \varrho_{\varepsilon}\right), \quad \varrho_{k}=\min (k, \varrho), \quad \Omega_{k, \varepsilon}:=\left\{\varrho_{\varepsilon}<k\right\}, \quad \Omega_{k}:=\{\varrho<k\} .
$$

Как и в теореме 2.1, для почти всех $\tau$ выполнено (2.5), что дает интегрируемость $\varrho(\tau, \cdot) \ln \varrho(\tau, \cdot)$ на $\mathbf{R}^{d}$. Для всех $\delta>0$ и $\tau \in(\delta, 1)$ с указанным 
свойством имеем равенство

$$
\begin{aligned}
\int_{\delta}^{\tau} \int_{\mathbf{R}^{d}}\left(\partial_{t} \varrho_{\varepsilon}\right)\left(\ln \varrho_{k, \varepsilon}\right) \zeta_{j}^{2} d x d t= & -\int_{\delta}^{\tau} \int_{\mathbf{R}^{d}}\left(a^{i k} \partial_{x_{i}} \varrho\right) * w_{\varepsilon} \frac{\partial_{x_{k}} \varrho_{\varepsilon}}{\varrho_{\varepsilon}} \zeta_{j}^{2} I_{\Omega_{k, \varepsilon}} d x d t \\
& -2 \int_{\delta}^{\tau} \int_{\mathbf{R}^{d}}\left(a^{i k} \partial_{x_{i}} \varrho\right) * w_{\varepsilon} \partial_{x_{k}} \zeta_{j}\left(\ln \varrho_{k, \varepsilon}\right) \zeta_{j} d x d t \\
& +\int_{\delta}^{\tau} \int_{\mathbf{R}^{d}} I_{\Omega_{k, \varepsilon}}\left(\left(b_{0} \varrho\right) * w_{\varepsilon}, \frac{\nabla \varrho_{\varepsilon}}{\varrho_{\varepsilon}}\right) \zeta_{j}^{2} d x d t \\
& \left.+2 \int_{\delta}^{\tau} \int_{\mathbf{R}^{d}}\left(\left(b_{0} \varrho\right) * w_{\varepsilon}, \nabla \zeta_{j}\right)\right) \zeta_{j} \ln \varrho_{k, \varepsilon} d x d t
\end{aligned}
$$

Поскольку $\varrho_{\varepsilon} \partial_{t} \ln \varrho_{k, \varepsilon}=\partial_{t} \varrho_{k, \varepsilon}$, левая часть равна

$$
\begin{aligned}
E(j, k, \varepsilon, \delta):= & \int_{\mathbf{R}^{d}} \zeta_{j}^{2}(x) \varrho_{\varepsilon}(\tau, x) \ln \varrho_{k, \varepsilon}(\tau, x) d x \\
& -\int_{\mathbf{R}^{d}} \zeta_{j}^{2}(x) \varrho_{\varepsilon}(\delta, x) \ln \varrho_{k, \varepsilon}(\delta, x) d x \\
& +\int_{\mathbf{R}^{d}} \zeta_{j}^{2}(x) \varrho_{k, \varepsilon}(\delta, x) d x-\int_{\mathbf{R}^{d}} \zeta_{j}^{2}(x) \varrho_{k, \varepsilon}(\tau, x) d x .
\end{aligned}
$$

Сохраняя $\delta>0$ фиксированным, полагая $\varepsilon \rightarrow 0$ и используя интегрируемость функции $t \mapsto\|\varrho(t, \cdot)\|_{W^{p, 1}\left(B_{2 j}\right)}$ на $[\delta, \tau]$, а также непрерывность и строгую положительность $\varrho$ на $[\delta, \tau] \times B_{2 j}$, получаем

$$
\begin{aligned}
S_{j, k, \delta}:= & \int_{\delta}^{\tau} \int_{\mathbf{R}^{d}}\left(A \nabla \varrho, \frac{\nabla \varrho}{\varrho}\right) \zeta_{j}^{2} I_{\Omega_{k}} d x d t \\
= & -2 \int_{\delta}^{\tau} \int_{\mathbf{R}^{d}}\left(A \nabla \varrho, \nabla \zeta_{j}\right) \zeta_{j} \ln \varrho_{k} d x d t+\int_{\delta}^{\tau} \int_{\mathbf{R}^{d}} I_{\Omega_{k}}\left(b_{0}, \frac{\nabla \varrho}{\varrho}\right) \zeta_{j}^{2} \varrho d x d t \\
& +2 \int_{\delta}^{\tau} \int_{\mathbf{R}^{d}}\left(b_{0}, \nabla \zeta_{j}\right) \zeta_{j}\left(\ln \varrho_{k}\right) \varrho d x d t-E(j, k, \delta)
\end{aligned}
$$

где

$$
\begin{aligned}
E(j, k, \delta):= & \int_{\mathbf{R}^{d}} \zeta_{j}^{2}(x) \varrho(\tau, x) \ln \varrho_{k}(\tau, x) d x-\int_{\mathbf{R}^{d}} \zeta_{j}^{2}(x) \varrho(\delta, x) \ln \varrho_{k}(\delta, x) d x \\
& +\int_{\mathbf{R}^{d}} \zeta_{j}^{2}(x) \varrho_{k}(\delta, x) d x-\int_{\mathbf{R}^{d}} \zeta_{j}^{2}(x) \varrho_{k}(\tau, x) d x .
\end{aligned}
$$

Интегрируя по частям в интеграле от

$$
\left(A \nabla \varrho, \nabla \zeta_{j}\right) \zeta_{j} \ln \varrho_{k}=\left(\nabla \varrho, \zeta_{j} A \nabla \zeta_{j}\right) \ln \varrho_{k}
$$

и записывая $\left(b_{0}, \nabla \varrho\right)=\left(A^{-1 / 2} b_{0}, A^{1 / 2} \nabla \varrho\right)$, находим

$$
\begin{aligned}
S_{j, k, \delta}= & 2 \int_{\delta}^{\tau} \int_{\Omega_{k}}\left(\frac{\nabla \varrho}{\varrho}, A \nabla \zeta_{j}\right) \zeta_{j} \varrho d x d t+2 \int_{\delta}^{\tau} \int_{\mathbf{R}^{d}} \operatorname{div}\left(\zeta_{j} A \nabla \zeta_{j}\right)\left(\ln \varrho_{k}\right) \varrho d x d t \\
& +\int_{\delta}^{\tau} \int_{\Omega_{k}}\left(b_{0}, \frac{\nabla \varrho}{\varrho}\right) \zeta_{j}^{2} \varrho d x d t+2 \int_{\delta}^{\tau} \int_{\mathbf{R}^{d}}\left(b_{0}, \nabla \zeta_{j}\right) \zeta_{j}\left(\ln \varrho_{k}\right) \varrho d x d t \\
& -E(j, k, \delta) \leqslant \sqrt{S_{j, k, \delta}}\left(2\left\|I_{\Omega_{k}} \sqrt{A} \nabla \zeta_{j}\right\|_{L^{2}(\mu)}+\left\|A^{-1 / 2} b_{0}\right\|_{L^{2}(\mu)}\right) \\
& +R_{j, k, \delta}-E(j, k, \delta),
\end{aligned}
$$


где

$$
\begin{aligned}
R_{j, k, \delta}:= & 2 \int_{\delta}^{\tau} \int_{\mathbf{R}^{d}} \operatorname{div}\left(\zeta_{j} A \nabla \zeta_{j}\right)\left(\ln \varrho_{k}\right) \varrho d x d t \\
& +2 \int_{\delta}^{\tau} \int_{\mathbf{R}^{d}}\left(b_{0}, \nabla \zeta_{j}\right) \zeta_{j}\left(\ln \varrho_{k}\right) \varrho d x d t
\end{aligned}
$$

Поскольку функция $\varrho_{0}$ гёльдерова на $B_{2 j}$, имеем $\lim _{\delta \rightarrow 0} \varrho(\delta, x)=\varrho(0, x)$ равномерно по $B_{2 j}$ (см., например, теоремы 7.1 и 10.1 в гл. III в [16]). Значит, $\lim _{\delta \rightarrow 0} E(j, k, \delta)=E(j, k, 0)$. Следовательно, (2.14) выполнено для $\delta=0$. Сохраняя $k$ фиксированным, заметим, что для заданного $\varepsilon>0$ для всех достаточно больших чисел $j$ вида $j=r_{l}$ с числами $r_{l} \rightarrow \infty$, выбранными согласно (2.13), величина $R_{j, k, \delta}$ может быть сделана меньшей $\varepsilon$ по модулю. В самом деле, из предположений и оценок

$$
\sup _{x}\left|\nabla \zeta_{j}(x)\right| \leqslant j^{-1} \sup _{x}|\nabla \zeta(x)|, \quad \sup _{x}\left|\partial_{x_{i}} \partial_{x_{m}} \zeta_{j}(x)\right| \leqslant j^{-2} \sup _{x}\left|\partial_{x_{i}} \partial_{x_{m}} \zeta(x)\right|
$$

вытекает, что для всех $j=r_{l}$ первый член в выражении для $R_{j, k, \delta}$ может быть оценен через

$$
\begin{aligned}
& M\left\|\ln \varrho_{k}\right\|_{L^{2}(\mu)} r_{l}^{-2}\left(\int_{0}^{1} \int_{\left\{r_{l} \leqslant|x| \leqslant 2 r_{l}\right\}}\|A(t, x)\|^{2} \mu_{t}(d x) d t\right)^{1 / 2} \\
& \quad+M\left\|\ln \varrho_{k}\right\|_{L^{2}(\mu)} r_{l}^{-1}\left(\int_{0}^{1} \int_{\left\{r_{l} \leqslant|x| \leqslant 2 r_{l}\right\}} \Theta_{A}(t, x)^{2} \mu_{t}(d x) d t\right)^{1 / 2},
\end{aligned}
$$

где $M$ - постоянная, зависящая от максимума первых и вторых производных $\zeta$. Тот факт, что $\ln \varrho_{k} \in L^{2}(\mu)$, вытекает из $\mu$-интегрируемости $|\ln (|x|+1)|^{2}$, так как на множестве $\{x: \varrho(t, x) \leqslant 1\}$ имеем $|\ln \varrho(t, x)|^{2} \sqrt{\varrho(t, x)} \leqslant C$ и потому

$$
|\ln \varrho(t, x)|^{2} \varrho(t, x) \leqslant(2 d+2)^{2}(\ln (|x|+1))^{2} \varrho(t, x)+C(|x|+1)^{-d-1} .
$$

Аналогично, ввиду неравенства Коши и оценки

$$
|b(t, x)| \leqslant\left\|A^{1 / 2}(t, x)\right\|\left|A^{-1 / 2}(t, x) b(t, x)\right|,
$$

второй член в выражении для $R_{j, k, \delta}$ мажорируется величиной

$$
M r_{l}^{-1}\left\|A^{-1 / 2} b\right\|_{L^{2}(\mu)}\left\|\ln \varrho_{k}\right\|_{L^{4}(\mu)}^{2}\left(\int_{0}^{1} \int_{r_{l} \leqslant|x| \leqslant 2 r_{l}}\|A(t, x)\|^{2} \mu_{t}(d x) d t\right)^{1 / 2} .
$$

Величины $E(j, k, 0)$ ограничены снизу постоянной, не зависящей от $j$ и $k$, ибо мы рассматриваем такие $\tau$, что $\varrho(\tau, \cdot) \ln \varrho(\tau, \cdot) \in L^{1}\left(\mathbf{R}^{d}\right)$, причем $\varrho(0, \cdot) \ln \varrho(0, \cdot) \in L^{1}\left(\mathbf{R}^{d}\right)$ по условию. Это означает, что интегралы от $|\sqrt{A} \nabla \varrho / \varrho|^{2}$ по множествам $\Omega_{k}$ относительно $\mu$ равномерно ограничены. Полагая $k \rightarrow \infty$, а затем $j \rightarrow \infty$, мы заключаем, что функция $|\sqrt{A} \nabla \varrho|^{2} \varrho^{-1}$ интегрируема по $[0, \tau] \times \mathbf{R}^{d}$. Кроме того, ввиду $(2.14)$ для ее интеграла $S$ выполнено неравенство $S \leqslant \sqrt{S}\left\|A^{-1 / 2} b_{0}\right\|_{L^{2}(\mu)}+E$, 
где $E$ - разность энтропий $\varrho(0, \cdot)$ и $\varrho(\tau, \cdot)$. Это дает желаемую оценку. Теорема 2.2 доказана.

3 а м е ч а н и е (. 2.2) Если $A$ равномерно ограничено, то предположение, что $\Lambda \in L^{4}(\mu)$ во второй теореме, можно ослабить до включения $\Lambda \in L^{2}(\mu)$.

\section{3. Более высокая интегрируемость и ограниченность плотностей}

Результаты предыдущего раздела показывают, что решения глобально интегрируемы в некоторой степени больше 1. Здесь мы выведем еще более сильные свойства интегрируемости, а также глобальной ограниченности при дополнительных предположениях о коэффициентах. Ниже мы считаем, что мера $\mu$ задана плотностью $\varrho$, причем при каждом $t \in[0,1)$ функция $x \mapsto \varrho(t, x)$ является вероятностной плотностью относительно меры Лебега.

Положим $\|u\|_{p, q, \tau}=\left\|u I_{[0, \tau]}\right\|_{p, q}$, где $t \rightarrow I_{[0, \tau]}(t)$ - индикатор отрезка $[0, \tau]$.

Лемма 3.1. Пусть $d>2$. Для всякой Функиии $u \in \mathbf{H}^{2,2}([0, \tau] \times$ $\left.\mathbf{R}^{d}\right) \cap L^{2, \infty}\left([0, \tau] \times \mathbf{R}^{d}\right)$ справедливо неравенство

$$
\|u\|_{p, q, \tau} \leqslant c(d, p)\left(\|\nabla u\|_{L^{2}\left([0, \tau] \times \mathbf{R}^{d}\right)}+\|u\|_{2, \infty, \tau}\right),
$$

где $2 \leqslant q, 2<p \leqslant 2 d /(d-2), 1 / q+d /(2 p)=-d / 4$.

Д ок аз а т ель с т в о. Пусть $\delta=d / 2-d / p=2 / q$. Тогда

$$
\frac{(d-2) \delta}{2 d}+\frac{1-\delta}{2}=\frac{1}{p}
$$

Пусть $r=2 d(p \delta(d-2))^{-1}$. Тогда $r \geqslant 1(r>1$ при $p<2 d /(d-2))$ и $r^{\prime}=r(r-1)^{-1}=2(p(1-\delta))^{-1}$. Записав $|u|^{p}=|u|^{p \delta}|u|^{p(1-\delta)}$ и применив неравенство Гёльдера с $r$ и $r^{\prime}$, получим

$$
\left(\int_{\mathbf{R}^{d}}|u|^{p} d x\right)^{1 / p} \leqslant\left(\int_{\mathbf{R}^{d}}|u|^{2 d /(d-2)} d x\right)^{(d-2) \delta / d}\left(\int_{\mathbf{R}^{d}}|u|^{2} d x\right)^{(1-\delta) / 2} .
$$

Неравенство Соболева дает

$$
\|u(t, \cdot)\|_{p} \leqslant c(d, p)\|\nabla u(t, \cdot)\|_{2}^{\delta}\|u(t, \cdot)\|_{2}^{1-\delta} .
$$

Следовательно, для почти всех $t \in[0, \tau]$ имеем

$$
\|u(t, \cdot)\|_{p} \leqslant c(d, p)\left(\|\nabla u(t, \cdot)\|_{2}\right)^{\delta}\left(\|u\|_{2, \infty, \tau}\right)^{1-\delta} .
$$

Поскольку $\delta=2 / q$, то

$$
\left(\int_{0}^{\tau}\|u(t, \cdot)\|_{p}^{q} d t\right)^{1 / q} \leqslant c(d, p)\left(\int_{0}^{\tau}\|\nabla u(t, \cdot)\|_{2}^{2} d t\right)^{1 / q}\left(\|u\|_{2, \infty, \tau}\right)^{1-2 / q} .
$$

В силу неравенства Юнга получаем требуемую оценку. Лемма 3.1 доказана. 
Лемма 3.2. Предположим, что выполнено условие (i) теоремы 2.1, причем дополнительно имеем

$$
\sup _{t \in[0,1]}\|b(t, \cdot)\|_{L^{s}\left(\mu_{t}\right)}<\infty, \quad \varrho \in L^{k s /(s-2)+1, k+(s-2) / s}\left([0, T] \times \mathbf{R}^{d}\right)
$$

с некоторьми $T \in[0,1], s>2, k \geqslant 2 / s$. Кроме того, пусть $\mu_{0}=$ $\varrho(0, \cdot) d x$, где $\varrho(0, \cdot) \in L^{k+1}\left(\mathbf{R}^{d}\right)$. Тогда для почти всех $\tau \in[0, T]$ имеем $\varrho(\tau, \cdot) \in W_{\text {loc }}^{1,1} u$

$$
\begin{aligned}
& \frac{2}{\alpha k(k+1)} \int_{\mathbf{R}^{d}} \varrho(\tau, x)^{k+1} d x+\int_{0}^{\tau} \int_{\mathbf{R}^{d}}|\nabla \varrho(t, x)|^{2} \varrho(t, x)^{k-1} d x d t \\
& \leqslant C(\alpha, \lambda, d, s) \int_{0}^{\tau}\left(\int_{\mathbf{R}^{d}} \varrho(t, x)^{k s /(s-2)+1} d x\right)^{(s-2) / s} d t \\
& \quad+\frac{2}{\alpha k(k+1)} \int_{\mathbf{R}^{d}} \varrho(0, x)^{k+1} d x
\end{aligned}
$$

где $\alpha$-константа из условия $A(t, x) \geqslant \alpha \cdot I, a C(\alpha, \lambda, d, s)$ - некоторое число, зависящее только от $\alpha, \lambda, d, s$.

Если же вместо (3.1) выполнено условие

$$
|b| \in L^{s}(\mu), \quad \varrho \in L^{k s /(s-2)+1}\left([0, T] \times \mathbf{R}^{d}\right),
$$

где $s>2, k>0$, то для почти всех $\tau \in[0, T]$ справедливо неравенство

$$
\begin{aligned}
& \frac{2}{\alpha k(k+1)} \int_{\mathbf{R}^{d}} \varrho(\tau, x)^{k+1} d x+\int_{0}^{\tau} \int_{\mathbf{R}^{d}}|\nabla \varrho(t, x)|^{2} \varrho(t, x)^{k-1} d x d t \\
& \leqslant C(\alpha, \lambda, d, s)\left(\int_{0}^{\tau} \int_{\mathbf{R}^{d}} \varrho(t, x)^{k s /(s-2)+1} d x d t\right)^{(s-2) / s} \\
& \quad+\frac{2}{\alpha k(k+1)} \int_{\mathbf{R}^{d}} \varrho(0, x)^{k+1} d x .
\end{aligned}
$$

Д оказательств о. Пусть $\varrho_{\varepsilon}-$ те же самые, что и в теореме 2.1 , в частности $\varepsilon \in\{1 / n\}$. В силу (2.3) имеем

$$
\int_{0}^{\tau} \int_{\mathbf{R}^{d}} \partial_{t}\left(\varrho * w_{\varepsilon}\right) \varphi d x d t=\int_{0}^{\tau} \int_{\mathbf{R}^{d}}\left[\left(a^{i j} \varrho\right) * \partial_{x_{i}} \partial_{x_{j}} w_{\varepsilon}-\left(b^{i} \varrho\right) * \partial_{x_{i}} w_{\varepsilon}\right] \varphi d x d t
$$

для всякой ограниченной измеримой функции $\varphi$ на $[0,1] \times \mathbf{R}^{d}$, ибо указанные свертки интегрируемы. Возьмем $\varphi(t, x):=\varrho_{\varepsilon}(t, x)^{k}$. Заметим, чTO

$$
\left[\left(a^{i j} \varrho\right) * \partial_{x_{j}} w_{\varepsilon}-\left(b^{i} \varrho\right) * w_{\varepsilon}\right] \varrho_{\varepsilon}^{k-1} \partial_{x_{i}} \varrho_{\varepsilon} \in L^{1}\left([0,1] \times \mathbf{R}^{d}\right) .
$$

Действительно, функция $\varrho_{\varepsilon}^{k}$ ограничена, а функции

$$
\left[\left(a^{i j} \varrho\right) * \partial_{x_{j}} w_{\varepsilon}-\left(b^{i} \varrho\right) * w_{\varepsilon}\right] \varrho_{\varepsilon}^{-1 / 2}, \quad\left|\nabla \varrho_{\varepsilon}\right| \varrho_{\varepsilon}^{-1 / 2}
$$

входят в $L^{2}\left([0,1] \times \mathbf{R}^{d}\right)$, как уже отмечалось в доказательстве теоремы 2.1. Следовательно, мы можем проинтегрировать по частям, что 
дает

$\int_{0}^{\tau} \int_{\mathbf{R}^{d}} \partial_{t}\left(\varrho * w_{\varepsilon}\right) \varrho_{\varepsilon}^{k} d x d t=-\int_{0}^{\tau} \int_{\mathbf{R}^{d}}\left[\left(a^{i j} \varrho\right) * \partial_{x_{j}} w_{\varepsilon}-\left(b^{i} \varrho\right) * w_{\varepsilon}\right] k \varrho_{\varepsilon}^{k-1} \partial_{x_{i}} \varrho_{\varepsilon} d x d t$.

Обозначим левую часть этого равенства через $L_{\varepsilon}$. Тогда

$$
L_{\varepsilon}=\frac{1}{k+1} \int_{\mathbf{R}^{d}} \varrho_{\varepsilon}(\tau, x)^{k+1} d x-\frac{1}{k+1} \int_{\mathbf{R}^{d}} \varrho_{\varepsilon}(0, x)^{k+1} d x
$$

Отметим, что интегрируемость $\varrho_{\varepsilon}(\tau, x)^{k+1}$ по $x$ следует из ограниченности этой функции и интегрируемости при $k=0$. Используя неравенство Гёльдера, оценим $L_{\varepsilon}$ снизу следующим образом:

$$
L_{\varepsilon} \geqslant \frac{1}{k+1} \int_{\mathbf{R}^{d}} \varrho_{\varepsilon}(\tau, x)^{k+1} d x-\frac{1}{k+1} \int_{\mathbf{R}^{d}} \varrho(0, x)^{k+1} d x .
$$

Рассмотрим правую часть $R_{\varepsilon}$ равенства (3.5). Используя равенство (2.9), получаем

$$
\begin{aligned}
R_{\varepsilon}= & -k \int_{0}^{\tau} \int_{\mathbf{R}^{d}} a^{i j} \partial_{x_{j}} \varrho_{\varepsilon} \partial_{x_{i}} \varrho_{\varepsilon} \varrho_{\varepsilon}^{k-1} d x d t \\
& -k \int_{0}^{\tau} \int_{\mathbf{R}^{d}}\left(\partial_{x_{i}} \varrho_{\varepsilon}(t, x) \varrho_{\varepsilon}(t, x)^{k-1} \int_{\mathbf{R}^{d}} \partial_{x_{j}} w_{\varepsilon}(x-y)\left[a^{i j}(t, y)-a^{i j}(t, x)\right]\right. \\
& \times \varrho(t, y) d y) d x d t \\
& +k \int_{0}^{\tau} \int_{\mathbf{R}^{d}} \varrho_{\varepsilon} \partial_{x_{i}} \varrho_{\varepsilon} \varrho_{\varepsilon}^{k-1}\left[\left(b^{i} \varrho\right) * w_{\varepsilon}\right] d x d t .
\end{aligned}
$$

Значит, в силу (2.10) (напомним, что при выводе (2.10) не использовалась $\mu$-интегрируемость $|\ln (1+|x|)|^{2}$ и существование энтропии $\left.\mu_{0}\right)$ имеем

$$
\begin{aligned}
R_{\varepsilon} \leqslant & -k \alpha \int_{0}^{\tau} \int_{\mathbf{R}^{d}}\left|\nabla \varrho_{\varepsilon}\right|^{2} \varrho_{\varepsilon}^{k-1} d x d t+k d^{3 / 2} \lambda\left(\int_{0}^{\tau} \int_{\mathbf{R}^{d}}\left|\nabla \varrho_{\varepsilon}\right|^{2} \varrho_{\varepsilon}^{k-1} d x d t\right)^{1 / 2} \\
& \times\left(\int_{0}^{\tau} \int_{\mathbf{R}^{d}} \varrho_{\varepsilon}^{k-1}\left(\varrho * q_{\varepsilon}\right)^{2} d x d t\right)^{1 / 2}+k\left(\int_{0}^{\tau} \int_{\mathbf{R}^{d}}\left|\nabla \varrho_{\varepsilon}\right|^{2} \varrho_{\varepsilon}^{k-1} d x d t\right)^{1 / 2} \\
& \times\left(\int_{0}^{\tau} \int_{\mathbf{R}^{d}} \varrho_{\varepsilon}^{k-1} \sum_{i=1}^{d}\left[\left(b^{i} \varrho\right) * w_{\varepsilon}\right]^{2} d x d t\right)^{1 / 2},
\end{aligned}
$$

где $q_{\varepsilon}$ - те же, что и в (2.10). Ввиду неравенства $a b \leqslant \alpha a^{2} / 4+b^{2} / \alpha$ получаем оценку

$$
\begin{aligned}
R_{\varepsilon} \leqslant & -\frac{1}{2} k \alpha \int_{0}^{\tau} \int_{\mathbf{R}^{d}}\left|\nabla \varrho_{\varepsilon}\right|^{2} \varrho_{\varepsilon}^{k-1} d x d t+\frac{k d^{3} \lambda^{2}}{\alpha} \int_{0}^{\tau} \int_{\mathbf{R}^{d}} \varrho_{\varepsilon}^{k-1}\left(\varrho * q_{\varepsilon}\right)^{2} d x d t \\
& +\frac{k}{\alpha} \int_{0}^{\tau} \int_{\mathbf{R}^{d}} \varrho_{\varepsilon}^{k-1} \sum_{i=1}^{d}\left[\left(b^{i} \varrho\right) * w_{\varepsilon}\right]^{2} d x d t .
\end{aligned}
$$


Соединяя оценки на $L_{\varepsilon}$ и $R_{\varepsilon}$, приходим к неравенству

$$
\begin{gathered}
\frac{2}{\alpha k(k+1)} \int_{\mathbf{R}^{d}} \varrho_{\varepsilon}(\tau, x)^{k+1} d x+\int_{0}^{\tau} \int_{\mathbf{R}^{d}}\left|\nabla \varrho_{\varepsilon}\right|^{2} \varrho_{\varepsilon}^{k-1} d x d t \\
\leqslant C(\alpha, \lambda, d)\left(\int_{0}^{\tau} \int_{\mathbf{R}^{d}} \varrho_{\varepsilon}^{k-1}\left(\varrho * q_{\varepsilon}\right)^{2} d x d t\right. \\
\left.\quad+\int_{0}^{\tau} \int_{\mathbf{R}^{d}} \varrho_{\varepsilon}^{k-1} \sum_{i=1}^{d}\left[\left(b^{i} \varrho\right) * w_{\varepsilon}\right]^{2} d x d t\right) \\
+\frac{2}{\alpha k(k+1)} \int_{\mathbf{R}^{d}} \varrho(0, x)^{k+1} d x .
\end{gathered}
$$

Заметим, что $\left|\left(b^{i} \varrho\right) * w_{\varepsilon}\right|^{2} \leqslant \varrho_{\varepsilon}\left(\left|b^{i}\right|^{2} \varrho\right) * w_{\varepsilon}$. По неравенству Гёльдера имеем

$$
\begin{aligned}
& \int_{\mathbf{R}^{d}} \varrho_{\varepsilon}^{k-1}\left|\left(b^{i} \varrho\right) * w_{\varepsilon}\right|^{2}(t, x) d x \leqslant \int_{\mathbf{R}^{d}} \varrho_{\varepsilon}^{k}\left(\left|b^{i}\right|^{2} \varrho\right) * w_{\varepsilon}(t, x) d x \\
& =\int_{\mathbf{R}^{d}}\left(\int_{\mathbf{R}^{d}} \varrho_{\varepsilon}(t, x)^{k} w_{\varepsilon}(x-y) d x\right)\left|b^{i}(t, y)\right|^{2} \varrho(t, y) d y \\
& \leqslant\left\|\left(b^{i}(t, \cdot)\right)^{2}\right\|_{L^{s / 2}\left(\mu_{t}\right)}\left(\int_{\mathbf{R}^{d}}\left(\int_{\mathbf{R}^{d}} \varrho_{\varepsilon}(t, x)^{k} w_{\varepsilon}(x-y) d x\right)^{s /(s-2)}\right. \\
& \left.\times \varrho^{(s-2) / s}(t, y) d y\right)^{(s-2) / s} \\
& \left.\leqslant\left\|b^{i}(t, \cdot)\right\|_{L^{s}\left(\mu_{t}\right)}^{2}\left(\int_{\mathbf{R}^{d}} \varrho_{\varepsilon}(t, x)^{k(s-2) / s} w_{\varepsilon}(x-y) d x\right) \varrho(t, y) d y\right)^{(s-2) / s} \\
& =\left\|b^{i}(t, \cdot)\right\|_{L^{s}\left(\mu_{t}\right)}^{2}\left(\int_{\mathbf{R}^{d}} \varrho_{\varepsilon}(t, x)^{k s /(s-2)+1} d x\right)^{(x)}
\end{aligned}
$$

Аналогично с учетом оценки

$$
\left|\varrho * q_{\varepsilon}(t, x)\right|^{2} \leqslant \varrho_{\varepsilon}(t, x) \int_{\mathbf{R}^{d}} \varrho(t, y) \frac{|x-y|^{4}}{\varepsilon^{4}} w_{\varepsilon}(x-y) d y
$$

получаем

$$
\begin{aligned}
& \int_{\mathbf{R}^{d}} \varrho_{\varepsilon}^{k-1}\left(\varrho * q_{\varepsilon}\right)^{2}(t, x) d x \leqslant \int_{\mathbf{R}^{d}} \int_{\mathbf{R}^{d}} \varrho_{\varepsilon}(t, x)^{k} \varrho(t, y) \frac{|x-y|^{4}}{\varepsilon^{4}} w_{\varepsilon}(x-y) d x d y \\
& \leqslant \gamma(s) \int_{\mathbf{R}^{d}} \varrho(t, y)\left(\int_{\mathbf{R}^{d}} \varrho_{\varepsilon}(t, x)^{k s /(s-2)} w_{\varepsilon}(x-y) d x\right)^{s /(s-2)} d y \\
& \leqslant \gamma(s)\left(\int_{\mathbf{R}^{d}} \int_{\mathbf{R}^{d}} \varrho(t, y) \varrho_{\varepsilon}(t, x)^{k s /(s-2)} w_{\varepsilon}(x-y) d x d y\right)^{s /(s-2)} \\
& =\gamma(s)\left(\int_{\mathbf{R}^{d}} \varrho_{\varepsilon}(t, x)^{k s /(s-2)+1} d x\right)^{(s-2) / s},
\end{aligned}
$$

где

$$
\gamma(s):=\left(\int_{\mathbf{R}^{n}}|x|^{2 s} g(x) d x\right)^{2 / s}
$$


Положим

$$
B(s):=\max _{t \in[0,1]}\|b(t, \cdot)\|_{L^{s}\left(\mu_{t}\right)}^{2} .
$$

Окончательно получаем

$$
\begin{aligned}
& \frac{2}{\alpha k(k+1)} \int_{\mathbf{R}^{d}} \varrho_{\varepsilon}(\tau, x)^{k+1} d x+\int_{0}^{\tau} \int_{\mathbf{R}^{d}}\left|\nabla \varrho_{\varepsilon}\right|^{2} \varrho_{\varepsilon}^{k-1} d x d t \\
& \leqslant C(\alpha, \lambda, d)(\gamma(s)+B(s)) \int_{0}^{\tau}\left(\int_{\mathbf{R}^{d}} \varrho_{\varepsilon}(t, x)^{k s /(s-2)+1} d x\right)^{(s-2) / s} d t \\
& \quad+\frac{2}{\alpha k(k+1)} \int_{\mathbf{R}^{d}} \varrho(0, x)^{k+1} d x .
\end{aligned}
$$

Переходя к пределу при $\varepsilon \rightarrow 0$, получаем искомую оценку для почти всякого $\tau \in[0, T]$. Действительно, для почти всякого $\tau$ имеем $\lim _{\varepsilon \rightarrow 0} \varrho_{\varepsilon}(\tau, x)=\varrho(\tau, x)$ для почти всех $x$, а правая часть неравенства выше остается ограниченной при $\varepsilon \rightarrow 0$. Из этого следует, что $\varrho(\tau, \cdot) \in W_{\text {loc }}^{1,1}$ при почти всех $\tau$. Поэтому $\lim _{\varepsilon \rightarrow 0} \nabla \varrho_{\varepsilon}(\tau, x)=\nabla \varrho(\tau, x)$ для почти всех $x$, что по теореме Фату дает указанную оценку.

В случае условия (3.3) доказательство полностью повторяет предыдущие рассуждения за исключением оценки интеграла от $\varrho_{\varepsilon}^{k-1}\left|\left(b^{i} \varrho\right) * w_{\varepsilon}\right|^{2}$, для которого имеем

$$
\begin{gathered}
\int_{0}^{\tau} \int_{\mathbf{R}^{d}} \varrho_{\varepsilon}^{k-1}\left|\left(b^{i} \varrho\right) * w_{\varepsilon}\right|^{2} d x d t \leqslant \int_{0}^{\tau} \int_{\mathbf{R}^{d}} \varrho_{\varepsilon}^{k}\left(\left|b^{i}\right|^{2} \varrho\right) * w_{\varepsilon} d x d t \\
=\int_{0}^{\tau} \int_{\mathbf{R}^{d}}\left(\int_{\mathbf{R}^{d}} \varrho_{\varepsilon}(t, x)^{k} w_{\varepsilon}(x-y) d x\right)\left|b^{i}(t, y)\right|^{2} \varrho(t, y) d y d t \\
\leqslant\left\|\left(b^{i}\right)^{2}\right\|_{L^{s / 2}(\mu)}\left(\int_{0}^{\tau} \int_{\mathbf{R}^{d}}\left(\int_{\mathbf{R}^{d}} \varrho_{\varepsilon}(t, x)^{k} w_{\varepsilon}(x-y) d x\right)^{s /(s-2)}\right. \\
\times \varrho(t, y) d y d t)^{(s-2) / s} \\
\leqslant\|b\|_{L^{s}(\mu)}^{2}\left(\int_{0}^{\tau} \int_{\mathbf{R}^{d}} \varrho_{\varepsilon}(t, x)^{k s /(s-2)+1} d x d t\right)^{(s-2) / s}
\end{gathered}
$$

по неравенству Гёльдера. Лемма 3.2 доказана.

3 а м е ч а н и е 3.1. Из доказательства видно, что условие равенства единице интегралов от $\varrho(t, x)$ по $x$ можно заменить на условие равномерной ограниченности этих интегралов.

Теорема 3.1. Пусть при предположениях теоремь 2.1 мы дополнительно имеем

$$
\sup _{t \in[0,1]}\|b(t, \cdot)\|_{L^{d}\left(\mu_{t}\right)}<\infty
$$

и $\mu_{0}=\varrho(0, \cdot) d x$, где $\varrho(0, \cdot) \in L^{p}\left(\mathbf{R}^{d}\right)$ при всех $p \in[1,+\infty)$. Тогда $\varrho \in L^{p, q}\left([0, \tau] \times \mathbf{R}^{d}\right)$ для всех $p, q \in[1,+\infty) u \tau \in(0,1)$. 
Д ок а з а т е ль с тво. Рассмотрим случай $d>2$. Пусть $\varrho \in$ $L^{k s /(s-2)+1, k+(s-2) / s}\left([0, \tau] \times \mathbf{R}^{d}\right)$, где $k \geqslant 2 / s$ и $s>2$. Тогда в силу неравенства (3.2) имеем

$$
\begin{aligned}
& \int_{0}^{\tau} \int_{\mathbf{R}^{d}}|\nabla \varrho(t, x)|^{2} \varrho(t, x)^{k-1} d x d t \\
& \leqslant C(\alpha, \lambda, d, s) \int_{0}^{\tau}\left(\int_{\mathbf{R}^{d}} \varrho(t, x)^{k s /(s-2)+1} d x\right)^{(s-2) / s} d t \\
& \quad+\frac{2}{\alpha k(k+1)} \int_{\mathbf{R}^{d}} \varrho(0, x)^{k+1} d x .
\end{aligned}
$$

По теореме вложения Соболева, применяемой к функциям $x \mapsto$ $\varrho(t, x)^{(k+1) / 2} \mathrm{c}$

$$
\left|\nabla \varrho^{(k+1) / 2}\right|^{2}=\frac{(k+1)^{2}}{4}\left|\varrho^{(k-1) / 2} \nabla \varrho\right|^{2},
$$

имеем

$$
\begin{aligned}
& \frac{4}{(k+1)^{2}} \int_{0}^{\tau}\left(\int_{\mathbf{R}^{d}} \varrho(t, x)^{d(k+1) /(d-2)} d x\right)^{(d-2) / d} d t \\
& \leqslant \int_{0}^{\tau} \int_{\mathbf{R}^{d}}|\nabla \varrho(t, x)|^{2} \varrho(t, x)^{k-1} d x d t \\
& \leqslant \\
& \quad \widetilde{C}(\alpha, \lambda, d, s) \int_{0}^{\tau}\left(\int_{\mathbf{R}^{d}} \varrho(t, x)^{k s /(s-2)+1} d x\right)^{(s-2) / s} d t \\
& \quad+\frac{2}{\alpha k(k+1)} \int_{\mathbf{R}^{d}} \varrho(0, x)^{k+1} d x .
\end{aligned}
$$

Таким образом,

$$
\begin{aligned}
& \left(\|\varrho\|_{d(k+1) /(d-2), k+1, \tau}\right)^{k+1} \\
& \quad \leqslant C(\alpha, \lambda, d, s, k)\left(\|\varrho\|_{k s /(s-2)+1, k+(s-2) / s, \tau}\right)^{k+(s-2) / s}+M(k, \alpha),
\end{aligned}
$$

где

$$
M(k, \alpha):=\frac{k+1}{2 \alpha k} \int_{\mathbf{R}^{d}} \varrho(0, x)^{k+1} d x
$$

Теперь положим

$$
p_{n}:=p_{n-1}+\frac{2}{d-2}, \quad q_{n}:=q_{n-1}+\frac{2}{d}, \quad q_{1}=1, \quad p_{1}=\frac{d}{d-2} .
$$

По теореме 2.1 имеем $\varrho \in L^{p_{1}, 1}\left([0, \tau] \times \mathbf{R}^{d}\right)$ при всех $\tau<1$. Это дает возможность начать итерации с помощью (3.6). А именно, если в (3.6) положить $s=d$ и $k=q_{n-1}-(d-2) / d=q_{n}-1$, то придем к оценке

$$
\left(\|\varrho\|_{p_{n}, q_{n}, \tau}\right)^{q_{n}} \leqslant C\left(\alpha, \lambda, d, d, q_{n}-1\right)\left(\|\varrho\|_{p_{n-1}, q_{n-1}, \tau}\right)^{q_{n-1}}+M\left(q_{n}-1, \alpha\right) .
$$

Так как $p_{n} \rightarrow \infty$ и $q_{n} \rightarrow \infty$ при $n \rightarrow \infty$, то теорема доказана при $d>2$. Случаи $d=1$ и $d=2$ даже проще, поскольку в неравенстве Соболева 
вместо показателя $d /(d-2)$ можно брать любое число $r>1$. Однако эти случаи можно не рассматривать отдельно, а вывести из результата для $d=3$. Для этого от функции двух. переменных можно перейти к функции трех переменных $u=\varrho\left(t, x_{1}, x_{2}\right) g\left(x_{3}\right)$, где $g$ - стандартная гауссовская плотность. Мера $u d x d t$ удовлетворяет нашему уравнению на $[0,1) \times \mathbf{R}^{3}$ с коэффициентами $a^{i j}$ и $b^{i}$, совпадающими с прежними при $i, j \leqslant 2$, причем $a^{33}=1, a^{3 j}=a^{j 3}=0, b^{3}(t, x)=-x_{3}$. Теорема 3.1 доказана.

Теорема 3.2. Предположим, что при условиях теоремь 2.1 для некоторого числа $\beta>d+2$ мь имеем $|b| \in L^{\beta}(\mu)$ и $\varrho(0, \cdot) \in L^{\infty}\left(\mathbf{R}^{d}\right)$. Пусть либо

$$
\sup _{t \in[0,1]}\|b(t, \cdot)\|_{L^{d}\left(\mu_{t}\right)}<\infty
$$

либо $\varrho \in L^{p}\left([0, \tau] \times \mathbf{R}^{d}\right)$ при всех $\tau<1$ и некотором $p>1$. Тогда $\varrho \in L^{\infty}\left([0, \tau] \times \mathbf{R}^{d}\right)$ для всякого $\tau<1$.

Д о к а з а т ел ь с т в о. Пусть $d>2$. Зафиксируем $\tau<1$. Пусть $\varrho \in L^{k \beta /(\beta-2)+1}\left([0, \tau] \times \mathbf{R}^{d}\right)$, где $k>0$. Тогда в силу (3.4) получаем

$$
\begin{aligned}
& \int_{0}^{\tau} \int_{\mathbf{R}^{d}}|\nabla \varrho(t, x)|^{2} \varrho(t, x)^{k-1} d x d t \\
& \leqslant \\
& \quad C(\alpha, \lambda, d, \beta)\left(\int_{0}^{\tau} \int_{\mathbf{R}^{d}} \varrho(t, x)^{k \beta /(\beta-2)+1} d x d t\right)^{(\beta-2) / \beta} \\
& \quad+\frac{2}{\alpha k(k+1)} \int_{\mathbf{R}^{d}} \varrho(0, x)^{k+1} d x
\end{aligned}
$$

Следовательно,

$$
\begin{aligned}
& \frac{4}{(k+1)^{2}}\left(\left\|\nabla\left(\varrho^{(k+1) / 2}\right)\right\|_{L^{2}\left([0, \tau] \times \mathbf{R}^{d}\right)}\right)^{2} \\
& \quad \leqslant C(\alpha, \lambda, d, \beta)\left(\int_{0}^{\tau} \int_{\mathbf{R}^{d}} \varrho(t, x)^{k \beta /(\beta-2)+1} d x d t\right)^{(\beta-2) / \beta} \\
& \quad+\frac{2}{\alpha k(k+1)} \int_{\mathbf{R}^{d}} \varrho(0, x)^{k+1} d x .
\end{aligned}
$$

В силу неравенства (3.4) для почти всех $t<\tau$ имеем

$$
\begin{aligned}
\int_{\mathbf{R}^{d}} \varrho(t, x)^{k+1} d x \leqslant & k(k+1) C(\alpha, \lambda, d, \beta)\left(\int_{0}^{\tau} \int_{\mathbf{R}^{i}} \varrho^{k \beta /(\beta-2)+1} d x d t\right)^{(\beta-2) / \beta} \\
& +\int_{\mathbf{R}^{d}} \varrho(0, x)^{k+1} d x
\end{aligned}
$$

откуда получаем

$$
\begin{aligned}
\left(\left\|\varrho^{(k+1) / 2}\right\|_{2, \infty, \tau}\right)^{2} \leqslant & k(k+1) C(\alpha, \lambda, d, \beta)\left(\int_{0}^{\tau} \int_{\mathbf{R}^{d}} \varrho^{k \beta /(\beta-2)+1} d x d t\right)^{(\beta-2) / \beta} \\
& +\int_{\mathbf{R}^{d}} \varrho(0, x)^{k+1} d x
\end{aligned}
$$


Лемма 3.1 для $u=\varrho^{(k+1) / 2}$ при $p=q=2(d+2) / d$ дает оценку $\left\|\varrho^{(k+1) / 2}\right\|_{p, q, \tau} \leqslant C\left(d, \frac{2(d+2)}{d}\right)\left(\left\|\nabla\left(\varrho^{(k+1) / 2}\right)\right\|_{L^{2}\left([0, \tau] \times \mathbf{R}^{d}\right)}+\left\|\varrho^{(k+1) / 2}\right\|_{2, \infty, \tau}\right)$. Используя неравенство $(a+b)^{2} \leqslant 2 a^{2}+2 b^{2}$ и найденные выше оценки, получаем

$$
\begin{aligned}
\left(\|\varrho\|_{L^{(d+2)(k+1) / d}\left([0, \tau] \times \mathbf{R}^{d}\right)}\right) & k+1 \\
\leqslant & 4(k+1)^{2} C(\alpha, \lambda, d, \beta)\left(\|\varrho\|_{L^{k \beta /(\beta-2)+1}\left([0, \tau] \times \mathbf{R}^{d}\right)}\right)^{k+(\beta-2) / \beta} \\
& +\left(2+\frac{k+1}{\alpha k}\right) \int_{\mathbf{R}^{d}} \varrho(0, x)^{k+1} d x .
\end{aligned}
$$

Так как $\varrho(0, \cdot) \in L^{\infty}\left(\mathbf{R}^{d}\right)$, то сушествует такая константа $C$, что

$$
\left(2+\frac{k+1}{\alpha k}\right) \int_{\mathbf{R}^{d}} \varrho(0, x)^{k+1} d x \leqslant C^{k+1} \quad \forall k \geqslant 1 .
$$

Теперь положим

$$
\begin{aligned}
& p_{n}=\frac{(d+2)(\beta-2)}{d \beta}\left(p_{n-1}+\frac{2}{\beta-2}\right), \quad p_{1}=\frac{d}{d-2}, \\
& A_{n}=\|\varrho\|_{L^{p_{n}}\left([0, \tau] \times \mathbf{R}^{d}\right)}, \quad C_{1}=4\left(\frac{d}{d+2}\right)^{2} C(\alpha, \lambda, d, \beta) .
\end{aligned}
$$

Мы имеем $\varrho \in L^{p_{1}}\left([0, \tau] \times \mathbf{R}^{d}\right)$ в силу теоремы 3.1 , если выполнено условие на $b(t, \cdot)$, а если $\rho \in L^{p}$ с $p>1$, то это же выводится из (3.7). Заметим, что

$$
1<\frac{(d+2)(\beta-2)}{d \beta} \leqslant \frac{d}{d-2}
$$

ибо $\beta>d+2$. Поэтому $\left.p_{n} \geqslant((d+2) \beta-2) /(d \beta)\right)^{n}$. Для доказательства принадлежности $\varrho$ к пространству $L^{\infty}\left([0, \tau] \times \mathbf{R}^{d}\right)$ достаточно установить равномерную ограниченность $\left\{A_{n}\right\}$. Предположим, что $A_{n} \rightarrow \infty$. Тогда существует такое $N$, что для всякого $n>N$ имеем

$$
-\frac{C}{A_{n-1}}<1, \quad\left(A_{n-1}\right)^{-2 / \beta}<1, \quad\left(\frac{C}{A_{n-1}}\right)^{p_{n} d /(d+2)}<1 .
$$

Пусть $k:=p_{n} d /(d+2)-1$. Тогда

$$
p_{n-1}=\frac{k \beta}{\beta-2}+1, \quad p_{n-1} \frac{\beta-2}{\beta}-p_{n} \frac{d}{d+2}=-\frac{2}{\beta}
$$

и потому из (3.7) получаем

$$
\left(A_{n}\right)^{p_{n} d /(d+2)} \leqslant p_{n}^{2} C_{1}\left(A_{n-1}\right)^{p_{n-1}(\beta-2) / \beta}+C^{p_{n} d /(d+2)} .
$$

Следовательно,

$$
\left(\frac{A_{n}}{A_{n-1}}\right)^{p_{n} d /(d+2)} \leqslant p_{n}^{2} C_{1}\left(A_{n-1}\right)^{-2 / \beta}+\left(\frac{C}{A_{n-1}}\right)^{p_{n} d /(d+2)} \leqslant p_{n}^{2} C_{1}+1
$$


откуда получаем

$$
\ln A_{n}-\ln A_{n-1} \leqslant \frac{d+2}{p_{n} d} \ln \left(p_{n}^{2} C_{1}+1\right), \quad n>N
$$

Поскольку $p_{n} \geqslant((d+2)(\beta-2) /(d \beta))^{n}$, то полученная выше оценка дает сходимость ряда из $\ln A_{n}-\ln A_{n-1}$, что противоречит неограниченности $A_{n}$. Случай $d \leqslant 2$ обосновывается так же, как и в предыдущей теореме. Теорема 3.2 доказана.

3 а м е ч а н и е 3.2 . (i) Согласно сказанному в замечании 2.1, в теоремах 3.1 и 3.2 можно не требовать интегрируемости функции $\varrho(0, x) \ln \varrho(0, x)$, ибо интегрируемость $\varrho(0, x) \max (0, \ln \varrho(0, x))$ следует из включения $\varrho(0, \cdot) \in L^{p}\left(\mathbf{R}^{d}\right)$ с $p>1$.

(ii) Из доказательства и замечания 3.1 видно, что в теоремах 3.1 и 3.2 условие равенства единице интегралов от $\varrho(t, x)$ по $x$ можно заменить на условие равномерной ограниченности этих интегралов.

(iii) Отметим, что если в теореме 3.2 заранее дано, что $\varrho \in L^{p}([0, \tau] \times$ $\mathbf{R}^{d}$ ) при некотором $p>1$, то можно не требовать интегрируемости функции $|\ln (1+|x|)|^{2} \varrho(t, x)$, но ограниченность $\varrho(0, x)$ важна.

Теперь мы воспользуемся доказанной теоремой для получения оценок сверху для $\varrho$. Как и в эллиптическом случае, рассмотренном в работах [8]-[10], идея такова: чтобы получить поточечную оценку $\varrho(t, x) \leqslant \Phi(t, x)^{-1}$, надо рассмотреть меру $\nu:=\Phi \cdot \mu$ и установить ограниченность ее плотности. Мы будем рассматривать функции $\Phi$, не зависящие от $t$. Если $\Phi$ имеет локально ограниченные производные первого и второго порядка, то мера $\nu$ удовлетворяет уравнению

$$
L^{*} \nu=\left(a^{i j} \partial_{x_{i}} \partial_{x_{j}} \Phi\right) \varrho+2 \partial_{x_{i}} \Phi \partial_{x_{j}}\left(a^{i j} \varrho\right)-b^{i} \partial_{x_{i}} \Phi \varrho=-L \Phi \cdot \varrho+2 \partial_{x_{j}}\left(a^{i j} \partial_{x_{i}} \Phi \varrho\right),
$$

понимаемому в том же смысле, что и (1.1).

Теорема 3.3. Пусть выполнень все предположения теоремь 3.2, u пусть задана такая функиия $\Phi \geqslant c>0$ на $\mathbf{R}^{d}$ с локально ограниченньим производньми второго порядка, что $\varrho(0, x) \leqslant C \Phi(x)^{-1}$, $\Phi \in L^{1}\left(\mu_{0}\right) u \Phi^{1+\varepsilon},|L \Phi|^{\beta / 2} \Phi^{1-\beta / 2},|A \nabla \Phi|^{\beta} \Phi^{1-\beta} \in L^{1}(\mu)$,

$$
\sup _{t \in[0,1]} \int_{\mathbf{R}^{d}} \Phi(x) \varrho(t, x) d x<\infty
$$

с некоторьм $\varepsilon>0$. Тогда для всякого $\tau<1$ найдется такое число $C_{\tau}$, чтоо $\varrho(t, x) \leqslant C_{\tau} \Phi(x)^{-1}$ для почти всех $(t, x) \in[0, \tau] \times \mathbf{R}^{d}$.

Д о к а з а т е л ь с т в о. Из рассуждений, использованных при доказательстве теоремы 3.2 , и замечания 3.2 видно, что достаточно установить оценку (3.4) с $s=\beta$ для меры $\nu=\Phi \cdot \mu$, плотность которой входит в $L^{1+\varepsilon}\left([0,1] \times \mathbf{R}^{d}\right)$ ввиду ограниченности $\varrho$. В этой оценке речь шла об однородном уравнении, причем меры $\mu_{t}$ были вероятностными. Однако 
при наложенных условиях такая же оценка остается в силе и при наличии указанной выше правой части, если вместо условия $\mu_{t}\left(\mathbf{R}^{d}\right)=1$ предполагать лишь равномерную ограниченность мер $\mu_{t}$. Действительно, в правой части (3.5) с $\nu$ вместо $\mu$, т.е. с $u:=\Phi \varrho$ вместо $\varrho$, дополнительно появится интеграл от выражения

$$
-(L \Phi \cdot \varrho) * w_{\varepsilon}\left[u * w_{\varepsilon}\right]^{k}+\left[2 \partial_{x_{j}}\left(a^{i j} \partial_{x_{i}} \Phi \varrho\right)\right] * w_{\varepsilon}\left[u * w_{\varepsilon}\right]^{k} .
$$

Положим $\xi:=L \Phi / \Phi, \eta:=|A \nabla \Phi| / \Phi$ и оценим этот интеграл $J$ следующим образом:

$$
\begin{aligned}
J= & -\int_{0}^{\tau} \int_{\mathbf{R}^{d}}(\xi u)_{\varepsilon} u_{\varepsilon}^{k} d x d t-2 k \int_{0}^{\tau} \int_{\mathbf{R}^{d}}\left(a^{i j} \partial_{x_{i}} \Phi \varrho\right)_{\varepsilon} u_{\varepsilon}^{k-1} \partial_{x_{j}} u_{\varepsilon} d x d t \\
\leqslant & \left(\int_{0}^{\tau} \int_{\mathbf{R}^{d}}|\xi|^{\beta / 2} u d x d t\right)^{2 / \beta}\left(\int_{0}^{\tau} \int_{\mathbf{R}^{d}} u_{\varepsilon}^{k \beta /(\beta-2)+1} d x d t\right)^{(\beta-2) / \beta} \\
& +2 k\left(\int_{0}^{\tau} \int_{\mathbf{R}^{d}}(\eta u)_{\varepsilon}^{2} u_{\varepsilon}^{k-1} d x d t\right)^{1 / 2}\left(\int_{0}^{\tau} \int_{\mathbf{R}^{d}}\left|\nabla u_{\varepsilon}\right|^{2} u_{\varepsilon}^{k-1} d x d t\right)^{1 / 2} .
\end{aligned}
$$

Остается заметить, что имеет место равенство

$$
\int_{0}^{\tau} \int_{\mathbf{R}^{d}}|\xi|^{\beta / 2} u d x d t=\int_{0}^{\tau} \int_{\mathbf{R}^{d}}|L \Phi|^{\beta / 2} \Phi^{1-\beta / 2} \varrho d x d t
$$

и неравенство

$$
\begin{aligned}
& \int_{0}^{\tau} \int_{\mathbf{R}^{d}}(\eta u)_{\varepsilon}^{2} u_{\varepsilon}^{k-1} d x d t \\
& \quad \leqslant\left(\int_{0}^{\tau} \int_{\mathbf{R}^{d}} \eta^{\beta} \Phi \varrho d x d t\right)^{2 / \beta}\left(\int_{0}^{\tau} \int_{\mathbf{R}^{d}} u_{\varepsilon}^{k \beta /(\beta-2)+1} d x d t\right)^{(\beta-2) / \beta}
\end{aligned}
$$

что проверяется таким же образом, как и в лемме 3.2. Теорема 3.2 доказана.

П р и м е р 3.1. Предположим, что $A$ и $A^{-1}$ равномерно ограничены, $A$ равномерно липшицево по $x$, причем для некоторых $\beta>d+2$, $r>0, \varepsilon>0, K>0$ имеем

$$
\begin{gathered}
|b| \in L^{\beta}(\mu), \quad \exp \left[(2 K+\varepsilon)|x|^{r}\right] \in L^{1}(\mu), \\
\sup _{t \in[0,1]} \int_{\mathbf{R}^{d}} \exp \left(K|x|^{r}\right) \varrho(t, x) d x<\infty .
\end{gathered}
$$

Пусть $\sup _{t \in[0,1]}\|b(t, \cdot)\|_{L^{d}\left(\mu_{t}\right)}<\infty$. Наконец, функция $\exp \left(K|x|^{r}\right) \varrho(0, x)$ ограничена и интегрируема по $\mathbf{R}^{d}$. Тогда для всякого $\tau<1$ найдется такое число $C(\tau)$, что $\varrho(t, x) \leqslant C(\tau) \exp \left(-K|x|^{r}\right),(t, x) \in[0, \tau] \times \mathbf{R}^{d}$. Для выполнения условия (3.8) и условий на $b$ и $\varrho(0, \cdot)$ достаточно оџенок $|b(t, x)| \leqslant C \exp \left(2 K \beta^{-1}|x|^{r}\right), \varrho(0, x) \leqslant C \exp \left(-K^{\prime}|x|^{r}\right)$ с $K^{\prime}>K$ и

$$
(x, b(t, x)) \leqslant c_{1}-c_{2}|x|^{r}, \quad c_{2}>2 r K \sup _{t, x}\|A(t, x)\| .
$$


Действительно, пусть $\Phi \in C^{2}\left(\mathbf{R}^{d}\right), \Phi(x)=\exp \left(K|x|^{r}\right)$ при $|x| \geqslant 1$. Все условия теоремы 3.3 выполнены. При условии $(3.9)$ выберем $\delta \in(0, \varepsilon)$ так, что выполнено неравенство $r(2 K+\delta) \sup _{t, x}\|A(t, x)\|<c_{2}$, и возьмем какую-нибудь функцию $V \in C^{2}\left(\mathbf{R}^{d}\right)$, равную $\exp \left[(2 K+\delta)|x|^{r}\right]$ при $|x| \geqslant 1$. Тогда при некотором $c$ справедлива оценка $L V \leqslant c$. Из [1] следует существование решения и равномерная ограниченность норм $\|V \varrho(t, \cdot)\|_{L^{1}\left(\mathbf{R}^{d}\right)}$. Выполнены также остальные условия теоремы 3.3. Аналогично при более слабых условиях можно получить степенную оценку.

Аналогичные теоремы справедливы в ситуации теоремы 2.2 .

Большая часть работы была выполнена во время визитов первого и третьего авторов в университет Билефельда.

\section{СПИСОК ЛИТЕРАТУРЫ}

1. Bogachev V.I., Da Prato G., Röckner M. Existence of solutions to weak parabolic equations for measures. - Proc. London Math. Soc., 2004, v. 88, № 3, p. 753-774.

2. Bogachev V.I., Da Prato G., Röckner M. On solvability of weak parabolic equations for measures. - Preprint. Bielefeld: Reseach Center BiBoS, Bielefeld University, 2005.

3. Bogachev V.I., Krylov N.V., Röckner M. On regularity of transition probabilities and invariant measures of singular diffusions under minimal conditions. - Comm. Partial Differential Equations, 2001, v. 26, №11-12, p. 2037-2080.

4. Stannat $W$. Time-dependent diffusion operators on $L^{1}$. - Preprint SFB 343 , № 00-080. Bielefeld: Univ. Bielefeld, 2000.

5. Bogachev V.I., Röckner M. Regularity of invariant measures on finite- and infinitedimensional spaces and applications. - J. Funct. Anal., 1995, v. 133, № 1, p. 168-223.

6. Bogachev V.I., Krylov N. V., Röckner M. Regularity of invariant measures: the case of non-constant diffusion part. - J. Funct. Anal., 1996, v. 138, № 1, p. 223-242.

7. Bogachev V.I., Röckner M., Wang F.-Y. Elliptic equations for invariant measures on finite and infinite dimensional manifolds. - J. Math. Pures Appl., 2001, v. 80, №2, p. 177-221.

8. Metafune G., Pallara D., Rhandi A. Global regularity of invariant measures. J. Funct. Anal., 2005, v. 223, № 2, p. 396-424.

9. Богачев В.И., Крылов Н. В., Рекнер М. Регулярность и глобальные оценки плотностей инвариантных мер диффузионных процессов. - Докл. РАН., 2005, т. 405, № 5 (в печати).

10. Bogachev V.I., Krylov N. V., Röckner M. Elliptic equations for measures: regularity and global bounds of densities. - Preprint № 05-06-186, Bielefeld: Research Center BiBoS, Bielefeld University; J. Math. Pures Appl. (to appear).

11. Carlen E. A. Conservative diffusions. - Comm. Math. Phys., 1984, v. 94, № 3, p. 293315.

12. Cattiaux P., Fradon M. Entropy, reversible diffusion processes, and Markov uniqueness. - J. Funct. Anal., 1996, v. 138, № 1, p. 243-272.

13. Cattiaux P., Léonard C. Minimization of the Kullback information of diffusion processes. - Ann. Inst. H. Poincaré, 1994, v. 30, № 1, p. 83-132; correction: ibid., 1995, v. 31, № 4, p. 705-707.

14. Föllmer H. Random fields and diffusion processes. - Lecture Notes in Math., 1988, v. 1362 , p. $101-203$.

15. Nelson E. Quantum Fluctuations. Princeton: Princeton Univ. Press, 1985, 147 p.

16. Ладыженская О. А., Солонников В. А., Уральчева Н. Н. Линейные и квазилинейные уравнения параболического типа. М.: Наука, 1967, 736 с.

Поступила в редакцию 31.X.2005 